Portland State University

PDXScholar

\title{
An Analysis of Oregon Youth Authority Populations: Who Receives Treatment and What Factors Influence Allocation of Treatment Resources?
}

Rebecca Arredondo Yazzie

Portland State University

Follow this and additional works at: https://pdxscholar.library.pdx.edu/open_access_etds

Part of the Criminology Commons, and the Social Work Commons Let us know how access to this document benefits you.

\section{Recommended Citation}

Yazzie, Rebecca Arredondo, "An Analysis of Oregon Youth Authority Populations: Who Receives Treatment and What Factors Influence Allocation of Treatment Resources?" (2017). Dissertations and Theses. Paper 3642.

https://doi.org/10.15760/etd.5526

This Dissertation is brought to you for free and open access. It has been accepted for inclusion in Dissertations and Theses by an authorized administrator of PDXScholar. Please contact us if we can make this document more accessible: pdxscholar@pdx.edu. 
An Analysis of Oregon Youth Authority Populations: Who Receives Treatment and What Factors Influence Allocation of Treatment Resources?

by

Rebecca Arredondo Yazzie

A dissertation presented in partial fulfillment of the requirements for the degree of

Doctor of Philosophy

in

Social Work and Social Research

Dissertation Committee:

William Feyerherm, Chair

Laura Nissen

Michael Taylor

Keith Kaufman

Melissa Thompson

Portland State University

2017 


\begin{abstract}
Service provision in both adult and juvenile correctional settings is an understudied phenomenon. Research has evaluated the use of evidence-based practices (EBP) in the treatment of mental health and substance abuse among adults and juveniles. Young, Farrell, Henderson and Taxman (2009) highlight the role of organizational factors including climate and culture, opportunities for staff training, resources, administrator attitudes and interagency collaboration in the application of EBP in correctional settings. The Oregon Youth Authority (OYA) aims to provide services to youth under the guise of EBP. Literature in the field of juvenile justice is limited in scope in areas of organizational factors, which are likely to influence the allocation of mental health treatment to youth. This study addressed this gap in existing research by using thematic analysis of focus group data with 28 OYA employees representing seven facilities. Data on 594 youth is offered to illustrate the variability of youth characteristics in OYA facilities. Facility data reflective of youth management and operations is presented to suggest context for staff observations and perceptions of how youth are identified for treatment. Results indicate staff knowledge about treatment and consistency in training along with frequency of organizational change affect climates in which treatment recommendations are made. Findings from this study will be beneficial to OYA as they attempt to meet the growing needs of mental health populations and address ongoing changes to agency staff, culture and climate. Implications from this study will contribute to literature on treatment service provision in juvenile correctional settings.
\end{abstract}




\section{Dedication}

To my parents, David and Pauline Arredondo, may this be a testament to all the sacrifices you have made to allow me to pursue my professional and educational endeavors. 


\section{Acknowledgments}

To my dissertation chair, Bill Feyerherm, thank you for youth relentless support, mentoring and guidance throughout this project.

To my dissertation committee, Drs. Keith Kaufman, Laura Nissen, Michael Taylor, and

Melissa Thompson, thank you for your encouragement, direction and support. For the late Dr. Jim Nash, who inspired my commitment to research.

To the research team at the Oregon Youth Authority, your consistent support and assistance in with this project is very much appreciated.

To my family and friends, brothers and sisters, and closest colleagues, thank you for fostering my passion for this project and sustaining perseverance alongside me. 


\section{Table of Contents}

Abstract

$\begin{array}{ll}\text { Dedication } & \text { ii }\end{array}$

Acknowledgements

List of Tables $\quad$ vi

Chapter One: Introduction 1

Background and Context 1

Evolution of Guiding Juvenile Justice Principles 3

From a Rehabilitative to Punitive Model 5

Chapter Two: Literature Review 9

Need for Research on Allocation of Treatment Resources $\quad 10$

$\begin{array}{ll}\text { Youth Factors } & 13\end{array}$

$\begin{array}{ll}\text { Staff Factors } & 17\end{array}$

$\begin{array}{ll}\text { Facility/Program and Organizational Factors } & 20\end{array}$

$\begin{array}{ll}\text { Theory } & 25\end{array}$

Critique of Existing Literature 29

Purpose and Significance of the Study 36

Research Questions 38

Variables to be included in the Study 42

Chapter Three: Methodology 44

$\begin{array}{ll}\text { Design } & 44\end{array}$

$\begin{array}{ll}\text { Participants } & 45\end{array}$ 
$\begin{array}{ll}\text { Procedures } & 51\end{array}$

Data Analysis $\quad 52$

Chapter Four: Results 56

$\begin{array}{ll}\text { Youth } & 56\end{array}$

$\begin{array}{ll}\text { Staff } & 62\end{array}$

$\begin{array}{ll}\text { Facility/Program } & 67\end{array}$

$\begin{array}{ll}\text { Chapter Five: Discussion } & 72\end{array}$

$\begin{array}{ll}\text { Study Implications } & 77\end{array}$

$\begin{array}{ll}\text { Limitations of the Study } & 80\end{array}$

Areas for Future Research $\quad 83$

$\begin{array}{ll}\text { References } & 85\end{array}$

$\begin{array}{ll}\text { Appendices } & 97\end{array}$

Appendix A: Case File Recording Form 97

Appendix B: Focus Group Script and Participant Survey 99 


\section{List of Tables}

Table 1: Descriptive Statistics for Participants 58

Table 2: Frequency of Participant Responses Using Likert 62

Scale Measurement

Table 3: Agency and Individual Facility Characteristics Across

68

Legislative Bienniums 


\section{Chapter One: Introduction}

\section{Background and Context}

Theorists and researchers in the social sciences have identified the need for further examination of referral patterns, service use, allocation of treatment resources, and treatment receipt among juvenile offenders in closed custody facilities. Social workers and mental health providers find themselves intersecting with juvenile and adult criminal justice systems as they work towards reintegrating incarcerated individuals into the community. As this occurs, issues of unequal access and disproportionality are revealed. Efforts to plan for transition back into the community often reveal availability and type of treatment resources for youth. Successful examination of these areas can assist both juvenile and adult justice systems serving multiple purposes, including accountability and rehabilitation.

The Juvenile Justice System (JJS) has become heavily populated with juvenile offenders in need of mental health treatment services. Approximately 600,000 youth are placed in detention facilities every year and close to 70,000 youth reside in juvenile correctional facilities every day (Mental Health and Juvenile Justice Collaboration, 2014). Currently, OYA has 566 youth in closed custody (OYA Quick Facts, 2017). In January 2011, during the onset of this study, there were approximately 800 youth in closed custody settings (OYA At a Glance, 2011). Among these youth are youth offenders who enter facilities with a mental health disorder and those who develop a disorder as a result of stressful environments in the facilities (Peterjl-Taykor, 1999). Commonly, these youth are diagnosed with Mood, Anxiety and Disruptive Behavior 
Disorders in conjunction with a coexisting Substance Abuse Disorder (Shelton, 1999). Current research also suggests that incarcerated youth experience high rates of exposure to trauma. As a result, they may suffer adverse effects and experience negative outcomes from these traumas (Coleman, 2005). Further, research is needed to appropriately develop mechanisms to ensure treatment service delivery is addressing negative outcomes for youth. Currently, there is no clear, objective, scientific measure to determine allocation of services after being indentified with a need for mental health treatment (Models for Change, 2006). Efforts such as the Models for Change initiative advocate for the use of clinical judgment, given the youth's diagnosis, level of impairment, and amenability to treatment in affording treatment resources to youth. This study aims to explore who is currently allocated mental health treatment within the Oregon Youth Authority and the factors influencing allocation.

The discussion begins with a brief history of the JJS to explain the evolution of its guiding principles. Second, following an overview of guiding principles is a summary of both rehabilitative and punitive models and their impact on providing treatment services to youth offenders. Third, a review of existing literature on youth, staff, and organizational factors and their influence on allocation of mental health treatment is presented. Fourth, social construction theory will be presented followed by an overview of the need for research on allocation of treatment resources in juvenile justice settings. Fifth, the purpose of this study and research questions studied are presented. Sixth, methods utilized in this study along with the study's results and implications will be 
highlighted. The next section will examine existing JJS values and the complexity of service delivery systems in correctional facilities.

Evolution of guiding principles. The first juvenile court was established in Cook County, Illinois, in 1899. According to many observers of the juvenile justice field, the founding ideals of the juvenile justice system remain strong within the system and need to be supported by all those committed to improving the lives of our children (Snyder \& Sickmund, 2006). The original orientation of the juvenile court was one that viewed juvenile offenders as abandoned, neglected, and poorly socialized youth in need of guidance (LeCroy, Stevenson, \& MacNeil, 2001). Placing minors under the jurisdiction of parens patriae, a term to describe the juvenile court system's doctrine of establishing the court as legal guardians or the state as parents (Snyder \& Sickmund, 2006), was an initial step of the juvenile court to ensure youth under the care of the court were properly dealt with. "Juvenile courts were meant to function 'in the best interests of the child,' and early juvenile correctional programs were supposed to be treatment programs rather than prisons" (Barton, 2006, p.49). This philosophy resulted from emphasizing treatment strategies over punitive strategies due to the rehabilitative potential of the juvenile offender. Over the course of decades, numerous Supreme Court decisions addressing concerns from victims of crime made the juvenile courts look more like criminal courts (Scott \& Steinerg, 2008). This is relevant in that many decisions have been made by the courts to interrupt the initial goal of bringing restoration to victims while still treating the youth as a juvenile. Beginning in the 1960s, numerous court decisions impacted juvenile courts in such a way they became more like its adult counterpart. With the emergence of 
"get tough" policies, many juvenile justice systems are adopting more harsh punitive models rather than rehabilitative models (Ashford, Sales, \& Reid, 2001).

In the mid 1990s, following many years of change, the concept of resiliency prompted discussion of risk and protective factors among adolescent offenders, and "knowledge of risk and protective factors found its way into mainstream juvenile justice policy" in regards to juveniles (Barton, 2006, p. 52). These initial changes included modifying ways juvenile offenders were handled in state courts. States made more attempts at balancing offender accountability, rehabilitation, and community protection (Scott \& Steinberg, 2008). The implementation of resiliency theory in assessment of mental health risk and protective factors was a significant contribution to policy formulated by OJJDP (Office of Juvenile Justice and Delinquency Prevention). The need to increase protective factors in youth where previous risk factors once existed was recognized by the JJS. The risk principle suggests level of service provided should match the level of risk to reoffend (Andrews \& Bonta, 2007). This recognition led to the development of programming to address risk factors in areas such as social, emotional, family, school, and community. Since the establishment of the OJJDP in 1974 juvenile justice policies were beginning to change and began addressing juvenile delinquency, such as use of boot camps and other nontraditional institution-based alternatives like diversion. Since the establishment of the first juvenile court in 1899, ongoing changes have caused both policy makers and service providers challenges in offering guidance and care to juvenile offenders under the original auspice of the JJS. These challenges include ensuring programs work towards rehabilitating the offender while supporting 
accountability for their crimes. These same challenges are even more prevalent when addressing youth with mental health needs in the JJS. Youth with mental health needs have unique aspects of rehabilitation and can often go unidentified. Due to this, policy makers have focused on addressing the inability of the JJS to identify the mental health needs of youth offenders and to provide treatment for them (Hertz, 2001).

Given the evolution of guiding principles and the emergence of the risk and protective framework, there is growing acknowledgment of mental health needs of youth in the JJS. However, the JJS has limited information to assess if and how often those needs are being met. Authors such as Hertz (2001) and Cocozza and Skowyra (2000) highlight the many obstacles present when the JJS attempts to address the mental health needs of youth in its care. One potential obstacle is the diminished likelihood that a youth will receive services when involved in two parallel systems (juvenile justice and mental health) unless a youth enters the mental health system via an alternative reason for entry (e.g. abuse or neglect). If a youth enters both the mental health and JJS system, there is potential for the youth to be transferred back and forth, with their needs unmet (Hertz, 2001). Furthermore, information is lacking on what services are provided in the JJS and the quality of such services (Cocozza \& Skowyra, 2000). The ongoing challenge is how to provide specialized mental health services within a juvenile correctional model.

From a rehabilitative to punitive model. Models adopted by the JJS over the years have influenced the decision to charge juvenile offenders as adults under harsher punitive frameworks. Leaving behind the rehabilitative model caused a period of transformation in relationship to policies and ways of handling juvenile offenders. In 
recent years, many states have moved towards a punitive model for juvenile justice policy (Snyder \& Sickmund, 2006). This model emphasizes the protection of the "public, public safety and promotion of the concept of punishment for criminal acts" (Snyder \& Sickmund, 2006, p.99) as its primary focus. This punitive model is a platform for "get tough" sentencing and statutory guidelines like those of Oregon's Measure Eleven. Measure Eleven was enacted by a voter initiative in 1995 . As a result, more youth have been placed on a long-term basis in juvenile facilities due to interests in separating juvenile serious offenders from adults in the adult correctional system. Measure Eleven has also led to extended facility stays for youth committed on person-to-person crimes. Wong (2001) highlights the increased stay of adult sex offenders given the new mandatory minimum sentences. As a result sex offenders have a disproportionate length of stay in comparison to those convicted of other crimes (Wong, 2001). Whether or not length of stay affects allocation of resources to address mental health needs of youth offenders is understudied.

A move from a rehabilitative to a punitive model contributes to the increase in offenders in facilities, due to its emphasis on accountability and support for incarceration (Ashford, Sales, \& Reid, 2001). Caeti, Cullens, Hemmens, and Burton (2003) studied organizational cultures of youth correctional systems and found, that "juvenile justice will likely face complications that derive from a mixture of conflicting philosophies between rehabilitation and punishment" (p. 386). Access to treatment, both while in custody and upon return to the community, is greatly affected as a result of these competing values. Often, youth offenders in need of treatment may end up in facilities 
due to a lack of community options. Opposing values also exist in treatment service provision in juvenile correctional settings. Different orientations in assessing "need" among juvenile offenders while in custody have triggered conversation in the fields of criminology. The RNR (Risk-Need-Responsivity) Model, a model commonly accepted in correctional management, dictates what correctional programming and treatment should be allocated to address risk factors of offenders (Andrews \& Bonta, 2007). This model asserts offenders have both dynamic (factors that can be influenced) and static (historical) risk factors. The risk principle explains the need to match level of service to offenders' risk to reoffend. Andrews and Bonta identify seven main areas of criminogenic needs. Criminogenic needs are thought to lead to criminal behavior. Some offenders have treatment needs requiring treatment, but not all of them are linked to criminal behavior. Those identified as major risks differ from those identified as minor risks. Having a diagnosed mental health disorder, low self-esteem, and vague feelings of personal distress are identified as minor risks. Correctional programming includes an array of correctional practices provided during incarceration, including evidenced based interventions and treatment activities which are designed to target an offender's risk to re-offend and serves the primary purpose of reducing recidivism. Some disagree with this primary purpose. Ashford, Sales, and Reid (2001) discuss the need for understanding why one chooses to offend versus simply addressing one's risk to re-offend. LeCroy, Stevenson, and MacNeil (2001) support the combination of two views of the youth offender: one that recognizes the youth in need of treatment and one that recognizes the need for punitive responses. Emerging from this acknowledgement is the rationale for the 
JJS to provide additional mental health treatment services to youth offenders in conjunction with correctional programming. This perspective can be summarized succinctly: when offending behavior is the result of maladaptive coping with mental health issues, then targeting the youth's ability to manage their own mental health symptoms may substantially reduce their risk to re-offend.

However, throughout the years it is apparent that juvenile correctional facilities have faced challenges in providing mental health treatment to offenders. These challenges include lack of funding streams for support systems available in the community, collaboration across multi-service systems (e.g. juvenile justice and mental health systems), and lack of mental health training for staff (Cocozza, 2000). As guiding principles and models adopted by the JJS have influenced the recognition to address the needs of youth offenders with mental health disorders, so does the evolving research and literature. The next section will provide an overview of relevant literature on youth factors, staff factors, and facility/program factors that have been found to influence the allocation of mental health treatment to individuals in care. 


\section{Chapter Two: Literature Review}

Treatment service delivery to youth in the JJS has been a significant focus of national attention within the last decade. During this time, studies examining screening and identification, assessment, and treatment outcomes have offered valuable information to those in the field. As previously discussed, the prevalence level for a diagnosable mental health disorder among all youth in the JJS is estimated to be as high as sixty-five to seventy percent (Shufelt \& Cocozza, 2006; Teplin, Abram, McClelland, Dulcan, \& Mericle, 2002; Wasserman \& McReynolds, 2004). Furthermore, youth in the JJS experience mental health disorders at a rate more than three times higher than youth in the general population (Mental Health and Juvenile Justice Collaboration for Change, 2014). With such high rates of prevalence, research has evolved into investigating referral patterns and receipt of treatment among youth.

Even after youth are identified with mental health diagnosis and/or in need of treatment, youth may remain at risk for having an unmet need for treatment (Rogers, Zima, Powell, and Pumariega, 2002). The risk for an unmet need for treatment can include not receiving treatment, not receiving the appropriate type of treatment, or not receiving adequate treatment. There is substantial evidence that factors aside from degree of need may play a role in determining which youth receive services. For example, evidence of race and ethnicity influencing mental health service referrals is supported by the work of Breda (2003) and Rogers, Zima, Powell, and Pumariega (2002). However, these studies focused on youth in the JJS in general and are not limited to youth placed in closed custody institutions. Few studies have included youth in closed custody 
institutional settings, which creates challenges in understanding allocation of resources and receipt of treatment unique to these settings like the OYA. OYA serves youth in community, camp, and closed custody institutional settings. It is likely youth in these settings are identified as in need of mental health treatment, but there may be vast variations in actual receipt of treatment.

\section{Need for Research on Allocation of Treatment Resources}

As recently as January 2001, one in ten young people experience severe mental illness (RAND Health, 2001). Despite this fact, fewer than twenty percent of these young people received treatment. Increased focus and attention has been given to young people with mental health issues living in general community settings as this concern continues to rise. Even more disturbing is the lack of attention to those youth who end up in closed custody institutions as a result of committing crimes. What is known is that over half of closed custody populations have been diagnosed with a mental health disorder. More efforts need to be made to determine how the decision making process is impacts the allocation of treatment to those identified with a need.

As previously mentioned, many youth who enter the JJS have a diagnosable mental health disorder. Ryan (2004) found prevalence of mood disorders such as depression and bipolar are more prevalent in the juvenile offender population than in the general adolescent population. Among those youth found to have a diagnosable mental health disorder, approximately twenty percent of are estimated to meet criteria for a serious mental health disorder (Cocozza \& Skowyra, 2000). Cocozza and Skowyra (2000) describe a "serious mental health disorder" as disorders describing diagnostic 
categories specific to schizophrenia, major depression, and bipolar disorder.

Additionally, rates of youth with co-occurring substance abuse disorders range from ten to twenty percent (Breda, 2003). Often, these youth are detained or placed in the JJS for minor offenses due to lack of community options (Skowyra \& Cocozza, 2006). Meeting the needs of youth offenders may be influenced by factors relating to competing values as well as facility, program, staff and youth characteristics. In addition to these factors is the pressure placed on the JJS to uphold public safety while spending fiscal resources conservatively.

As the literature supports the presence of youth offenders with mental health needs in correctional facilities, it also highlights an area that is understudied. An evaluation of how resources are allocated in the JJS would lend to a deeper understanding of the challenges the system faces given its dual purposes. Potential consequences of not investigating allocation of resources and treatment receipt include the absence of data on service gaps and inequalities. Given the diverse population the JJS serves, it is essential to identify which demographic groups (among those identified with a need) are receiving services. Other unintended consequences include enhancing correctional programming approaches without the exclusive knowledge regarding its impact on mental health treatment of offenders. Shelton (2005) highlights the continued limitation of juvenile offenders' rights to access mental health treatment as an integral part of their rehabilitation while in the JJS. If this continues, the prevalence of youth with unmet mental health needs will rise leaving a substantial burden on not only the JJS but also the 
systems of care in local communities that serve offenders transitioning into the community.

This literature review will first begin by summarizing the available literature (both empirical research and applicable theory) on treatment and allocation of resources to youth in custody. The approach used to explore literature on allocation of resources and receipt of treatment in closed custody settings such as OYA included a review of settings such as: community settings, medical case management settings, and organizational settings investigating factors which determine how individuals within the organization allocate resources to clients. This provides a framework by which this literature review is organized. It uses a perspective that divides decision-making factors into categories supported by the work of Fraser and Estabrooks (2008). Fraser and Estaborook's review was one of the first in the literature to examine factors in relation to resource allocation decisions in the nursing field. Health care fields including nursing and behavioral health serve populations from various demographics and client groups. Such large systems of delivery often share similar challenges in meeting client needs. As research is sparse on resource allocation decision-making processes in the juvenile justice field, concepts from these broader service delivery systems can be applied to systems like OYA. As a result of conducting a systematic literature review (meta analysis) of nursing case management, Fraser and Estabrooks four main groups of factors as significantly influencing decision making in regards to allocation of resources to clients in the various studies. They included case manager related factors, client related factors, information related factors, and system/program related factors. 
Using the evidence presented in the literature suggests the following in regards to the potential factors influencing allocation of resources to youth with treatment needs in closed custody settings within the larger JJS. From a systems perspective, factors such as how the organization frames "need" or "who is desirable" to receive treatment will impact who receives services. The decision-making process will include elements of youth factors (e.g. such as race and age), staff factors (e.g. perceptions about the youth) and program/facility factors (e.g. facility size) and will likely influence receipt of treatment.

\section{Status of the Literature}

Youth factors. Client factors such as race and ethnicity have been shown to influence referral to mental health treatment. Breda (2003) found no direct effects of ethnicity on referral for treatment, but when included with other factors such as offense type and prior record, ethnicity significantly affected the likeliness of a referral. Rogers, Zima, Powell and Pumariega (2002) highlight socio-demographic factors influencing treatment referral. Their study of 206 youth offenders residing in a California juvenile correctional facility found that not only a small proportion of youth received a referral to mental health services, but also that socio-demographic factors influenced referrals to treatment. Being female, Caucasian, and African American increased the likelihood of receiving a referral, whereas being Latino was related to no referral. Violent offenders received referrals and repeat offenders did not receive a referral. In a related study by Hertz (2001), youth gender and race influenced the decision to use mental health placement resources. Female and White offenders were more likely to receive placement 
resources than other groups (Herz, 2001). Interestingly, the interaction between race and gender was stronger than the direct effects of these variables by themselves. The Hertz study found White females were almost eight times more likely to be placed in mental health placements than Black males and almost two times more likely than White male and Black female offenders. The study found only a $4 \%$ probability of mental health placements overall, so these findings on the interaction of race and gender were very significant. Recently, a comprehensive literature review conducted by Spinney et al. (2016) examined twenty years of literature spanning the years of 1995 to 2014. Studies aimed at investigating the intersection of race and referral to mental health and substance abuse services in the JJS were included in the review. They found race effects in about $69 \%$ of the studies reviewed. This further supports earlier research in the area of race and demographics and the role they play in the referral process to various treatment services.

In looking at gender as a factor influencing treatment allocation decision making, Maschi, Schalbe, Morgen, Gibson, and Violette (2009) found significant differences in three areas of decision making for adolescent service needs: referral source, interventions, and factors for referral. In a study examining service utilization of adolescents in a suburban county, girls were more likely than males to be referred by school and outside practitioners. Males were more likely to be referred by court and parole entities. Interventions for males and females also were dissimilar. Males were more likely to have participated in out-of-home placements and drug and alcohol treatment. Females were more likely to have received interventions related to family planning. This study highlights gender as an important factor in service utilization. 
Gender as a factor in determining services may also be present in the process of allocating treatment resources by decision makers in OYA.

As gender is a factor of allocation of treatment to youth offenders, the discussion of treatment programs specifically designed for female offenders is of importance. As literature on "gender-specific" services emphasizes, girls have different pathways to offending and therefore require different programs and services than boys. Goals of the gender-responsive approach include program goals of empowerment and improving quality of life (Hubbard \& Matthews, 2008). Existing research supports the recognition that programs should be aware of and take gender differences into account as they provide treatment services to youth (Hubbard, 2007). In recent years, the OYA has adopted gender-specific programming, and it is essential to examine the allocation of treatment to females in custody to further investigate if this approach is being implemented equally to those who are identified with a need.

There is some evidence that youth factors, such as offense type, are related to allocation of treatment resources in court ordered treatment. Yan and Dannerbeck (2011) in a recent study found juvenile court judges in Missouri more likely to order mental health treatment for females than males. Females court ordered for treatment were less likely to have offense types other than sexual offenses. Males were less likely to be court ordered for mental health treatment if they had sexual and drug related offense types. They were more likely to receive a court order to participate in sexual offense specific and drug and alcohol treatment. Evidence in the study suggested judges might have considered offending behavior when determining treatment needs. Although both males 
and females did not significantly differ in mental health status, results indicated that offense type, age, and quantity of certain category offenses (public order or property offenses) influenced judges' orders for mental health treatment. The Oregon Youth Authority has a diverse population with respect to demographics, offense types, and mental health diagnosis. It would be beneficial to examine whether or not these findings translate to the OYA.

Other factors such as client problem behaviors have been found to influence allocation of resources. Although some studies are limited to community settings, they offer some explanatory basis for the decision making process when allocating resources. A study of 242 clients in a home care program found, among multiple predictors of case management resources, clients problem behaviors to be significant (Diwan, 1999). Client problem behaviors included non-compliance with medication or treatment and problems resulting from a mental health diagnosis. Additionally, new clients used case management resources more than existing clients. Diwan's study focused on the use of resources by the individuals in the sample but also measured the time spent with clients during the case as reported through interviews with case managers. Important aspects of decision-making were highlighted as a result of the study. They included the need for case managers to spend more time with clients to ensure the successful completion of a treatment plan. There are parallels from this study and its application to the OYA. Within OYA, youth behaviors are indicated by youth incident reports (YIRS). The allocation of resources and its relationship to client behaviors as evidenced by Diwan suggests youth in the OYA with a higher degree of YIRS (Youth Incident Reports) should be allocated 
more time and resources from case managers or monitors of youth treatment plans. In this setting, these individuals are mental health professionals.

Shelton (2005) also examined predictors of treatment provision among youth offenders in Maryland's detention and committed facilities. The study included a random sample $(\mathrm{N}=312)$ of youth, among them fifty percent of youth met criteria for a diagnostic classification for a mental health disorder. Shelton found in her analysis that a set of predictors including mental disorder, age, race, level of crime severity or number of incarceration episodes were predictive of the odds of receiving treatment. This study suggests that youth factors, similar to those found among OYA youth, can assist in determining how treatment resources are allocated to youth offenders while in custody.

Staff factors. Factors related to providers, as Fraser and Estabrooks (2008) found, are prominent in the allocation of resources to clients. Perceptions, judgment, and whether or not decision makers feel individuals are in a "desirable group" influence who is selected to receive resources. Leukemia and AIDS program patients were the topic of a study by Levin and Chapman (1993) which asked subjects in the sample to determine which programs clients with the two diagnosed diseases should be referred to. Results indicated the subjective decision of who would receive live saving- resources was attributed to perceptions and judgments of the subject in regards to who was more “deserving” of life-saving resources. When AIDS patients were negatively framed in the study method, subjects were likely to make the riskier decision of not allocating life saving resources. If the patients were positively framed, the reverse was found. This demonstration of the subjective view of who is "deserving" of treatment resources has 
not been explored in juvenile justice settings. Similarly, Taxman \& Bouffard (2003) found in a study of drug court counselors, treatment philosophy was related to treatment allocation rather than client needs. Although standardized screening instruments have been adopted in both the adult and juvenile justice systems, values held by staff or providers may influence allocation of treatment after initial identification of treatment need is made.

In a study investigating predictors of staff responses to youth behavior, Marsh \& Evans (2006) found demographics of juvenile justice staff to influence attitudes towards rehabilitation and treatment for youth offenders. Previous research by Mitchell et al., 2001; Moak \& Wallace, 2000 suggests that staff from minority groups are less likely to support punishment and tend to have a more favorable perception of delinquent youth. These perceptions among particular ethnic groups can play a role in determining treatment resources for youth offenders.

In another example of how attitudes and judgment influence allocation of resources, a study of 54 college students showed attitudes were significantly associated with decisions to allocate resources to treatment (Corrigan, Watson, Warpinksi, \& Gracia, 2004). Students exhibited a preference to mandate treatment using coercive means and segregation rather than rehabilitation for those with mental illness. Among those who displayed pity towards people with mental illness, as defined by Corrigan and colleagues, the decision to mandate treatment was directly associated with those feelings. This example illustrates how attitudes towards people with mental illness and beliefs about type of treatment can be connected. This finding is relevant to the OYA in that 
research has supported the link between correctional officer attitudes towards inmates with mental illness. Callahan (2004) found the stressful environment of correctional settings to be detrimental to inmates who were labeled "mentally ill." Labeling of target populations affected security staff and inmates alike. Many aspects of the study indicated that correctional officers' views of inmates mimicked those of the public. Moreover, Thompson, Newell, and Carlson (2016) investigated perceptions among parole officers (POs) in their understanding of how race and ethnicity affect treatment access of offenders in the community. The study was conducted in a county on the West Coast. POs reported difficulty in the ability to identify mental health needs and also mentioned the struggle with knowing if mental health symptoms were presented clinically. The convergence of non-mental health professionals and POs in the decision making process for treatment referral is an ongoing phenomenon in both the adult and juvenile system.

In examining suicidal behavior in correctional settings, Ivanoff and Hayes (2001) found correctional staffs' views of suicidal behavior and self-harm gestures to include the belief that inmates were "attention seeking." Due to this belief, officers felt behaviors should be ignored, inmates should not receive attention or intervention, and officers did not believe inmates were at risk for suicide. Although the American Correctional Association (1991) does not specifically detail training content or amount, it does recommend that officers receive training in areas of mental health crisis and suicide prevention. Training of correctional staff can be an important factor influencing allocation of mental health treatment in correctional settings. 
In an extensive literature review completed by Spinney et al. (2016) encompassing 20 years of literature investigating racial disparities in the JJS, emphasize one of many key points. The research exhibits the presence of a non-linear decision making model in the JJS, in regards to the intersection of mental health and justice. Interestingly, "drug treatment and mental health services may be offered in the context of detention programming or community-based programming, as an adjunct to probation, or as a component of long-term institutional programming" (Spinney et al., 2016, p.166). This finding further supports the need for cross training of staff, both correctional and mental health professionals to better identify and refer for services.

Although the OYA employs rehabilitative staff and not traditional correctional officers as in adult correctional settings, front line supervisory positions such as these are very similar according to the Group Life Coordinator (GLC) job description (OYA Career Opportunities, 2011). The interface of these supervisory staff with treatment providers in closed custody settings working with youth in need of treatment is inevitable. Providers' tendency to allocate resources to youth offenders may be subjected to influence from attitudes held by front line staff. Callahan (2004) also asserted cooperation among competing roles (clinical and security) is essential for mental health care to be effective in correctional settings. The OYA utilizes various positions to accomplish its mission of providing opportunities for reformation. There is a need to investigate whether those positions' attitudes influence allocation of resources.

Facility and program factors. At the organizational level or often at the program level there are value premises that have been found to influence allocation of resources. 
Duffee \& Carlson (2011) found that value premises such as deservingness, amenability to treatment, risk for social harm, and capacity of the supervising agency directly impacted allocation of drug treatment resources to probationers. Such value premises were highlighted in the review of cases from states with large waiting lists for drug treatment. They included New York, Michigan, Pennsylvania, and the District of Columbia. Although the review of case information and literature associated with these studies revolved around adult probationers, it can be applied to the value base that is demonstrated by the JJS. In recent years, many states have moved toward a punitive model for juvenile justice policy (Snyder \& Sickmund, 2006). This model emphasizes the protection of the "public and public safety and promotion of the concept of punishment for criminal acts" (Snyder \& Sickmund, 2006, p.99) as its primary focus. These shifts in focus will likely influence value premises such as whom is "deserving" and who is amenable to treatment. Given the research by Corrigan and colleagues, youth committing severe crimes or person-to-person crimes may not be viewed as "deserving" of mental health treatment depending on the providers' perceptions of them.

Among the most interesting research on organizational culture and climate and its effects on mental health care in the JJS is that of Gisson and Green (2006). In their study of effects of organizational climate and culture on access to mental health services for children in juvenile justice and child welfare systems, Glisson and Green (2006) found children who needed mental health services were much more likely to receive services when case managers belonged to more constructive work units, than those units with less constructive cultures. Their study of 588 children and 21 counties in Tennessee's juvenile 
justice system adds to the consideration of social context in the effectiveness of child welfare and juvenile justice systems. In particular, it relates to the influence of organizational culture on service outcomes. They cite three organizational areas that directly influence the way mental health resources are allocated and accessed. They are technological, strategic, and social factors. Technological factors include training of staff, and assessment by case management and counseling staff. Strategic factors include availability of resources, funding, and policies, which guide use. Social factors include expectations, norms, attitudes and perceptions that drive case management behavior and decision-making. Decision-making and allocation of treatment resources within a large bureaucratic system like the OYA can be highly influenced by these categories of factors. In a latest study, the relationship between leadership and organizational climate in working alliance with children in the child welfare system was examined. Findings suggested leadership and alliance with clients was mediated by culture, which in turn supports quality and effectiveness of care (Green, Albanese, Cafri, \& Aarons, 2014). Components from the work Glisson and Green will be explored in this study to determine if similar factors predict allocation of treatment with OYA's youth offender population.

Finally, in studying organizational context and its influence on the use of current best practices in a juvenile correctional agency, Farrell, J., Young, D., and Taxman, F. (2011) uncovered staff who had more favorable views of their supervisor and were less cynical about their office's ability to change were more likely to use best practice supervision practices. Focus group participants spoke to the type of leadership and observable organizational change as factors influencing youth identified for treatment. 
Use of best practices in the field of mental health on identification, screening, assessment and referral are likely to be influenced by such context in OYA offices and facilities.

Program factors including types of interventions offered have also been linked to service provision with youth with mental health needs. Programs including interventions targeted at parents, family, individuals and community systems have proven effective in reducing symptomology and recidivism (Pullman et al., 2006). Programming elements are equally important as facility location. Hertz (2001) found in a study of juvenile cases on the East coast, geographic location was significant in the use of mental health placements for offenders. Resource allocation to offenders in various regions was impacted by access to services and providers outside the JJS.

Finally, program factors such as agency resources, funding, and external systems of care may impact allocation of resources to youth with mental health treatment needs. Cocozza \& Skowyra (2000) found part of the challenge in addressing the high prevalence of youth with mental health needs in the JJS is the response of most jurisdictions towards the issue. Systems have simply increased numbers of secure beds available within local mental health systems and transferred youth back and forth between the two to address treatment needs (Cocozza \& Skowyra, p. 10). The system of care within the JJS is not equipped to deal with such needs of youth offenders. In addition to the system of care and whether or not youth offenders are being allocated resources is the issue of the funding stream. LeCroy, Stevenson, \& MacNeil (2001) assert major issues among agencies include the question of who is responsible for such specialized services for these youth, lack of systematic criteria used to identify those with needs, the high costs of services, 
and the increase in more punitive and less treatment-oriented modalities in the JJS. As a result, they are cautious to accept responsibility for youth with severe mental health issues. At the macro level, the amount of resources available to agencies, funding for services, and the process by which money follows the client impact whether or not individuals receive services. As OYA begins to address the needs of youth in their custody with mental health issues, it will be essential to examine the agency and determine if these previously stated factors impact how treatment resources are allocated at the various levels. OYA as a large agency has community programs, small camp settings, and larger closed custody institutional settings which may vary greatly in their makeup of fiscal, personnel, training, and support resources.

In summary, the literature highlights evidence that youth factors such as race, ethnicity, gender, offense type and severity, as well as exhibited behavior influence the allocation of treatment resources. In regards to staff, perceptions of which "is deserving" coupled with attitudes and judgments towards those in need of services have impact on the decision to allocate treatment resources just as staff race and ethnicity do. Finally, available research exists to support the notion that program value premises, culture and climate, and funding directly impact the allocation of treatment resources to those identified with a need.

Using the previously reviewed body of knowledge, this study will test a selected theory well known in the social sciences. In this study, social construction is used as a way to gain understanding about the complex reality of meeting the needs of youth in the JJS given guiding principles, history and various factors impacting the allocation of 
mental health treatment in the OYA. The tenets of social construction are reviewed in the following sections.

\section{Theory}

A very important tenet of social work theory is its approach in understanding social problems through the complex reality of the person-in-situation (Turner, 1986). As in the case of youth offenders, others who interact with them on a daily basis often shape stressors, which exist in their environment. Youth offenders who are in need of mental health treatment may be plagued by debilitating symptoms and are often at the hands of those providing care in the JJS. Therefore, the allocation of treatment resources and the decisions driving allocation warrant close examination. Due to the conflicting values in the JJS, referral and receipt of treatment services are largely impacted by individuals in positions of power, with impressions of the juvenile offender. Differences in referral and receipt can be explained through a social construction perspective. Social construction of target populations explained by Schneider and Ingram (1993) is a mechanism by which marginalized populations are adversely affected by policy decisions. These decisions are made with imposed labels and a social context framing marginalized groups as deviants or non-deserving in some aspect. Claims made by interested parties seek to explain an issue or problem (Miall, 1996). Some policy makers and providers in the JJS claim youth offenders should receive treatment targeted at reducing their risk of recidivating. Other claims makers may believe treatment of underlying causes of distress triggering criminal behavior should be a primary focus of the JJS. These competing claims influence the decision making process of allocating treatment resources to youth offenders. Although 
social construction theory is primarily applied in the political science and policy field, its application to the JJS is notable.

Social construction and its connection to settings like OYA are best described by Payne (1991). Knowledge that guides our behavior is understood as "reality" by those who obtain such knowledge. However, different views of "reality" are often experienced and thus, different conclusions exist. In regards to allocation of resources, decision makers are under the influence of a view of "reality" of offenders and their course of treatment and rehabilitation while in custody directly impacts various groups and populations. Then, with these understandings of "reality," social activity-in this case, the decision making process-becomes habitual (Payne, 1991). Once the process becomes habitual, the decision making process then becomes institutionalized. Given the large, complex, organizational structure of OYA, the process of decision-making and allocation of resources is vulnerable to institutionalization. Finally, once these decision-making mechanisms become legitimized, they attach meaning to the larger organized system. Elements of social construction are evident in both direct and indirect ways. The following paragraphs will provide examples of how social construction can impact youth treatment assignments given public perception, attitudes towards offenders, and views about mental illness. All of these concepts are likely to be found in settings like the OYA.

The social construction perspective proposes possible explanations for the differences in resource allocation in a number of ways. Despite evidence of the high prevalence of mental health diagnosis and treatment need, social constructions of offenders may be so widely shared and accepted they become rigid and laborious to 
disprove. Cocozza, Harstone, and Braff (1981) illustrate this by their discussion of violent juveniles as "bad" but also "mad." Their work on societal perceptions of violent juvenile offenders reveals how violent offenders are often viewed through various impressions and judgments throughout society. As stated previously by Diwan (1999), judges often use offense type as a basis for decision making of treatment court orders. Social constructions can be manipulated and used by policy proponents and public officials to convey an agenda (Schneider \& Ingram, 1993). Different messages are used to prioritize treatment and correctional programming in the JJS to reduce the likelihood juveniles will commit future crime. Juvenile offenders committed on violent offenses may be afforded treatment, but it may be treatment aimed at reducing aggression or cognitive distortions and not necessarily targeted at promoting management of specific symptoms of a mental health disorder. Administrators in the OYA participate in the decision making process for youth offenders as long as youth are in its custody. The social construction perspective is especially relevant to OYA as staff and policy makers influence how treatment resources are allocated to youth.

A great deal of literature has been written on the public perception of the offender. Although much of this literature is written on adults, it application to the OYA is useful, since OYA serves offenders ages twelve to twenty-five. Peterson and Palumbo (1997) found adult Oregonians participating in the Oregonians Support Alternatives Poll in 1995, perceived the term "prison inmate" as a violent, aggressive individual. When participants heard the word crime, they expressed notions relating to murder, robbery, and assault even though this type of misconduct makes up a small percentage of crimes 
committed by offenders. Despite different presentations of the terms, Oregonians displayed the same view of offenders. Scott and Steinberg (2008) asked eight hundred participants in a research study what should be the minimum age of transfer for a juvenile to adult court when a violent crime is committed. Their study highlighted the public's propensity to favor more lenient sanctions for younger offenders. An example of how media influences juvenile justice decision-making is the phenomena of school shootings. The social construction of an offender who may be experiencing a severe mental health disorder is demonstrated by the media's attention and focus on cases like the Virginia school shooting. When it became known that the perpetrator experienced symptoms of schizophrenia, increased public debate ensued. Mason and Mercer (1999) found as a result of their work, schizophrenics were arrested more often for crimes of violence than were other categories of mental illness. The public's perception of severely mentally ill offenders is often promoted into aspects of policing and social control. Naturally, those working within the OYA are susceptible to the same social and political influences that affect public perception. The potential for subtle (whether conscious or subconscious) display of social constructions is highly plausible given the nature of closed custody settings and youth offender client base. These subtle social constructions are found at various levels when decision makers allocate treatment resources to youth. They are described as "subtle" due to the unknown nature of how often they exist, when they exist, and by whom that are displayed. As outlined in this review, there is evidence that these social constructions occur when taking youth, staff, and facility/program factors into account. 
Given the previous section's description of social construction theory, a closer look at literature attempting to explain disparities in the allocation of mental health treatment is needed. The following section will review literature on established prevalence, plausible explanations for differences in allocation and offer a critical review of relevant studies as they relate to the need for further investigation into allocation of mental health treatment resources in the JJS. This will then be followed by an argument for the need for research in this area. The proposed study's aim seeks to add to the gap in the existing literature.

\section{Critique of Existing Literature}

Although there is sufficient information on prevalence rates of mental health disorders in the juvenile justice population, there is minimal information on allocation of treatment. Therefore, the available literature is limited to what is available throughout the human services and juvenile justice fields. A few of the studies included have limitations in their outdated origins. However, these studies demonstrate similarities in the provision of service in the JJS to other fields and are included to emphasize those similarities. This limitation is supported by Shelton (2005) who highlights the lack of studies exploring services available to incarcerated youth or utilization rates of this population in existing literature. Even more alarming is the lack of descriptors on which offenders receive mental health services while in custody. The following studies are offered to describe the growing need for further investigation.

Kelly, Macy and Mears (2005) estimated the gap between assessed needs and services among referrals to the Texas juvenile justice system. Using the Texas Juvenile 
Probation Commission Resource Survey 2000, they found a significantly large gap between referrals made with mental health or substance abuse needs and youth served. This finding highlights the importance of investigating through the use of future research why gaps exists in the JJS's service delivery model. Among the 14, 665 youth with an identified mental health need in two or more high-need areas, only 8,331 youth received services. Only an estimated $57 \%$ of those with needs received services. Additionally, among the substance abuse referrals, close to two-thirds of referrals did not receive nonresidential or residential treatment services. In comparison to subsequent studies mentioned in the following paragraphs, the Kelly, Macy, and Mears (2005) study does not include the use of a standardized mental health screening instrument which may limit the results presented. However, it does illustrate the need for further exploration in juvenile justice settings to determine if treatment resources are being allocated to those identified with a need. Given this study was limited to one state, exploring additional states in the United States would help to alleviate the gap in knowledge on treatment resource allocation.

In a study investigating detention admissions in Pennsylvania, 18, 607 youth were administered the Massachusetts Youth Screening Instrument Version 2 (MAYSI-2) (Cauffman, 2004). Results indicated $70 \%$ of males and $81 \%$ of females scored above clinical range cutoff in one of the domains (Alcohol/Drug Use, Angry-Irritable, Depressed-Anxious, Somatic Complaints, and/or Suicide Ideation). This study highlighted the availability of the MAYSI-2 instrument for further exploration of treatment resource allocation. This study was limited in that it did not measure treatment 
receipt after identification of those youth scoring in clinically significant ranges. Cauffman suggests the study's findings support the use of such a screen in reducing bias in allocation of treatment resources for the juvenile offender population. In another study, differences among those identified with a mental health diagnosis in their mental health service use including consultation only and treatment were found in a community sample of 760 children (Cohen, 1993). The study explored which demographic factors distinguished those youth who received mental health services from those who did not. The study found the amount of visits with a provider differed significantly for those youth 18-21 years of age versus those ages 11-17, those from middle income families versus those with greater than $\$ 50,000$ and low income families, and those residing in rural and semi-rural areas versus suburban, large towns or cities. Although this study explores resource allocation in a community sample, it provides evidence that youth demographics may influence access to treatment resources. This study provides a framework for a potential replication study in closed custody settings with youth offenders identified with a mental health diagnosis to investigate service use.

Teplin and colleagues (2005) investigated who received mental health services among a random sample of 1,829 juvenile detainees participating in the Northwestern Juvenile Project. Among those detainees who were found to have major mental health disorders, and some level of functional impairment, $15.4 \%$ received treatment in a juvenile detention center and $8.1 \%$ received treatment in the community. Of those detainees who were detected and treated, numerous predictors were found to be significant. Those included race/ethnicity, age, legal status, and reported treatment 
history. Interestingly, the study found non-Hispanic whites were two times more likely than racial/ethnic minorities to have been detected for a mental health disorder and almost one and a half more times to be treated. Younger detainees (ages 10-13 years of age) were more likely than older detainees (14 years and older) to be treated. Those detained processed as juveniles versus those processed as an adult were more likely to be treated as those who reported a previous treatment history. Limitations of this study include the use of a single site for the study and the impact on generalizability to other urban juvenile detention centers as well as the likelihood the true rate of service provision may be underreported due to the conservative definition of treatment need outlined by the research study. These results suggest a level of disparity among juvenile detainees with a mental health disorder across aspects of race/ethnicity, age, and legal status. Youth factors such as these are very relevant to the study of allocation of mental health treatment after initial identification of a mental health need through screening and assessment.

Researchers exploring racial differences in mental health service utilization in a study (Rawal, Romansky, Jenuwine, \& Lyons, 2004) including 473 participants who were petitioned, adjudicated, or incarcerated in Illinois' JJS found in comparison to African American and Hispanic youth, Caucasian youth had higher rates of prior, current, and overall service utilization rates. Among African American youth and Hispanic youth, Hispanic youth had the lowest rate of service utilization between the two while in the JJS. This study was limited to data from 1995-1996 and was retrospectively collected which may have potential issues with missing information gathered from existing charts. 
However, since there are relatively few studies on mental health service utilization in the JJS, it is valuable in its contribution to the field. Although various reasons were discussed in the study for possible explanations for the differing rates of service utilization, this study supports the need for additional examination of other state's juvenile justice systems to determine if similar patterns of utilization exist. The potential of this research will likely have implications on juvenile justice policy as more information will be revealed about access to services and characteristics of youth receiving services.

In another study examining service utilization and race/ethnicity, a multi-system study exploring five San Diego County public sectors (alcohol and drug abuse, child welfare, juvenile justice, mental health, and public schools) included a subsample of 1 , 256 youths to test for the presence of racial disparities. The study's results indicated differences in mental health service utilization across racial/ethnic groups even after other potentially influencing variables were controlled. Additionally, $79 \%$ on non-Hispanic white youths received services compared to $59 \%$ of Asian/American-Pacific Islanders. Non-Hispanic white youth also received more services compared to $64 \%$ of African Americans and $70 \%$ of Latinos. Although the study's focus included a large multi-system design, it demonstrates the presence of possible disparities in other large child welfare and juvenile justice agencies with similar high-risk youth profiles. One major limitation to this study included the study's reliance on parent and youth self-reports on service utilization. Also, reports of service use may also be biased to aspects of cultural bias. Despite these few limitations, this study further supports the existing research on youth factors, which may influence mental health service use and pattern of allocation. 
In a study of the Texas Department of Criminal Justice's Parole Division (Quinn, 2003), 28 of 66 field offices were targeted to determine parole officers' treatment orientation and patterns of prioritization of treatment resources (versus alternative services such as vocational and educational) for offenders on their caseload. Seniority, job type, and caseload size were significant in influencing prioritization of treatment resources. Additionally, respondents' race, size of city resided in, political ideology, gender, and education also influenced prioritization of treatment resources. One critique of this study includes the lack of information on the allocation of specific treatment resources to offenders. However, this study illustrates the significance of characteristics among decision makers, which influence the prioritization of treatment and potential allocation of such resources.

As Shelton (2005) provides information on youth factors influencing the provision of mental health treatment services, her work also illustrates the scarcity of resources given to youth after being diagnosed with a mental health disorder. In the secondary analysis Shelton found over half the sample (53\%) met criteria for a diagnosed mental disorder. Of those youth, only $26 \%$ received any treatment while being placed in Maryland's JJS. Although this study was aimed at exploring patterns of mental health services for offenders, it failed to highlight any possible reasons explaining this gap beyond the influence of race.

Atkins et al. (1999) sampled 75 youth from the South Carolina Department of Juvenile Justice to investigate the prevalence of psychopathology and level of symptomatology in comparison to various institutionalized youth samples. At least one 
diagnosis was found in $72 \%$ of incarcerated youth demonstrating prevalence of mental health symptoms in South Carolina's JJS. Limits of this study include the small sample from one select state in the region and the focus of the study was limited to prevalence rather than treatment received after identification. However, this study illustrates the need for further research into allocation of services given such high prevalence.

Finally, work by Pumariega et al. (1999) demonstrates the need for more investigation into allocation of mental health treatment resources in the JJS. Findings from this study build on findings from the Atkins study in that it illustrates differences in mental health service utilization among incarcerated youth and youth in the community. Using similar samples from the Atkins et al. (1999) study, incarcerated youths' utilization of mental health services was lower than comparison groups in the community both in community mental health centers and the state's inpatient residential program. A limitation to this study includes the measure of "prior" services or "service use history" to indicate utilization of mental health services. The comparison could have yielded more rich data on the limitations of mental health services in juvenile corrections settings if the utilization of services was measured during the time of incarceration or within the previous six months. This study further adds to the argument that more information is needed in regards to which youth are receiving services in the JJS and what factors influence allocation of those services. With this information, the JJS can begin to address gaps in services as well as increase utilization rates while youth are in closed custody facilities. 


\section{Purpose and Significance of the Study}

The purpose of this study is to examine mechanisms by which treatment resources are allocated to youth offenders in the OYA. Given the gap in existing research, this study aims to add to the existing literature on various factors influencing allocation of mental health treatment to youth in the JJS. The primary objective of this study is to determine which factors are strong predictors of treatment allocation. It will also determine, using tenets of social construction theory, whether subtle social constructions about youth offenders are related to treatment resource allocation. For the purposes of this study, mental health treatment is defined by any treatment aimed at reducing mental health symptoms. Treatment descriptions offered by agency and case records will inform classification of mental health treatment. Youth engagement is not a central focus of this study, rather it is whether or not treatment has been identified as a needed resource and allocated while youth are in the custody of OYA. Likewise, motivation to participate and amenability to mental health treatment are not primary in this proposed study. The opportunity for the OYA to uncover patterns of resource allocation is the primary rationale for this study. The results will afford OYA with data needed to make modifications or changes to existing policies, procedures, staff training, and current practices.

The following concepts are also provided for the purposes of understanding specific terms referenced in this study. The Juvenile Justice Information System (JJIS) is an electronic data system used by various partners within the Oregon JJS and all internal OYA employees. The Risk Needs Assessment (RNA) is an electronic risk needs 
assessment used by OYA to determine correctional case planning needs. The RNA includes eleven domains including: substance abuse mental health; education/school; use of free time; family/parenting; interpersonal relationships; criminal/delinquency history; employment; attitudes and beliefs; aggression and social skills. OYA field personnel (juvenile probation/parole officers) administer this within the first 30 days of probation. Similarly, within the first thirty days of closed custody commitment, facility staff administers the RNA. The RNA consists of a pre-screen and a full assessment. A full assessment is administered if the offender scores "high" on the pre-screen. The Multidisciplinary team (MDT) is the primary method of treatment planning while youth are under the custody of the OYA. These terms will be referenced throughout the methodology and data analysis sections. The next section will give a brief synopsis as to why this research is significant to the field of social work and juvenile justice.

There are many reasons why this study is significant to the field of social work and juvenile justice. The need to advocate for vulnerable populations is paramount. Established prevalence rates reveal many youth diagnosed with a mental health disorder are currently incarcerated and/or involved in the juvenile justice system at various levels (Boesky, 2001). Contributing to the field's knowledge of service delivery, resource allocation, and treatment receipt will aid policy makers, legislators, and administrators working within juvenile correctional to adequately meet mental health service needs of offenders in custody.

While social workers are often involved in discussions of social inequality, this study offers an opportunity to highlight factors, which lead to an unequal distribution of 
resources. Factors at multiple levels may influence allocation of resources in different ways. It is important to examine how this plays out in juvenile correctional facilities to address differences in how services are offered to incarcerated youth versus youth in the general population. Institutions providing services to youth offenders have an obligation to meet social welfare functions. When these are in question, it is the role of the social work student and researcher to pursue social justice in this arena. Due to a competing values framework in the JJS and multiple factors that influence allocation of treatment resources, youths who experience mental health symptoms may not receive adequate attention. If treatment is not adequate or the quality of care is not monitored and examined, the potential for dehumanizing or unfair treatment exists for this vulnerable population. Finally, administrators may assume fiscal expenditures are being spent in appropriate areas. Findings from this study will assist administrators in redistributing budgets to ensure fiscal resources are allocated to support the goal of addressing youth offenders' mental health needs. The next section will introduce the study's research questions and provide justifications for inclusion in this study.

\section{Research Questions}

This study explores factors that influence decisions to allocate resources to various youth offender groups. Factors such as youth, staff, and facility/program factors were investigated using a mixed methods methodology. The following three research questions are proposed:

1. What youth characteristics influence the odds of being assigned mental health treatment? 
1.1 What commitment offense characteristics influence the odds of receiving mental health treatment?

1.2 What demographic characteristics (including race/ethnicity, gender, and age) influence the odds of receiving mental health treatment?

1.3 What behavioral characteristics (Youth Incident Reports) influence the odds of receiving mental health treatment?

The following justifications are provided for inclusion of the first research question. Given the available research on youth, staff, and program factors, this research question offers an investigation as to whether or not findings in the literature are present within environments such as OYA. Specifically, commitment offense and behavior exhibited by offenders has been found to influence allocation of treatment in community settings such as residential programs. Testing for the occurrence of similar bias in closed custody settings will reveal substantial knowledge to those serving youth in OYA. Furthermore, work by Breda (2003), Rogers, Zima, Powell, and Pumariega (2002) support the need to explore whether or not race and ethnicity play a role in determining who receives treatment in the OYA. Findings from this research will be very important to those who wish to address racial disparities in treatment allocation among the juvenile justice population. Finally, this research question is essential to the study, as it will employ quantitative methods of data collection and analysis to achieve the mixed methods design of the study. The procedures used to examine this research question also call for a careful review of existing data about who is more likely to receive treatment resources given various factors. 
2. What factors does staff perceive as influencing the decision to allocate mental health treatment to youth offenders?

2.1 What youth factors does staff perceive as influencing the allocation of mental health treatment to youth offenders?

2.2 What staff factors does staff perceive as influencing the allocation of mental health treatment to youth offenders?

2.3 What facility/program or agency factors does staff perceive as influencing the allocation of mental health treatment to youth offenders?

2.4 How do youth, staff, and facility/program factors influence decisionmaking in the allocation process?

The second research question is included due a need to examine staff perceptions of how treatment resources are allocated to youth. As a mixed method design, this study uses both quantitative and qualitative methods to investigate research questions and this research question is included for the purposes of obtaining additional qualitative data. In the spirit of qualitative research, this question is included for the purposes of obtaining concepts and/or themes that will assist in understanding treatment allocation. This will also assist in revealing meanings attached to how factors influence allocation of treatment to youth. Since social construction theory is driving this study, this question will explore individuals' reality as they see it in OYA closed custody facilities. Since social interactions are complex and dynamic, as are large organizations, it is important to include this research question. It will provide an opportunity to investigate whether or not 
there is a presence of subtle social constructions that may be influencing allocation of treatment to youth.

This research question will address similar areas that will be investigated using strictly quantitative techniques to explore for similarities in meanings. Inclusion of this research question is central to the study's aim of investigating the potential factors that influence decisions among OYA staff to allocate mental health treatment resources.

3. Do the quantitative results and the qualitative findings about factors influencing the allocation of mental health treatment converge?

This research question is included due the mixed methods approach to investigating the research problem. Tashakkori and Creswell (2007) suggest inclusion of an explicit mixed methods question to specifically illustrate the intent to integrate the two sources of data. A thorough analysis of both quantitative and qualitative findings will help to attach meaning to an understudied area of juvenile justice literature.

As stated previously in the literature review, there are few studies that explore treatment allocation to youth with mental health needs in juvenile corrections settings. Exploring this research question will give more understanding to the convergence of data for discussion of implications to the field. This research question is especially important given the mixed methods design and will be needed for interpretation and conclusions of the study. In order to test all three research questions, specific methods from both a quantitative and qualitative approach were chosen for this study. Variables defined in the study will be described next followed by the methodology that will be used to collect information on each variable. 


\section{Variables Included in the Study}

Following the schema developed by Fraser and Estabrooks, there are three sets of independent variables included in this study. The first is a set of youth factors, starting with commitment offense. Commitment offense variable include a total of nine types of offenses ranging from criminal mischief to sex offense. The second is a set of demographics. Demographic variables include race/ethnicity, gender, and age. Race/ethnicity labels include White/Caucasian, African American, Hispanic, Native American, Asian, and Unknown/other. Gender variable labels include male and female. Age is included as a continuous variable. The third is a set of behavioral factors. Behavioral factors are indicated by number of youth incident reports (YIRs). Incidents of isolation are included as a variable as a second behavioral factor. Additional behavioral incidents such as suicide attempts are also included.

Other aspects of this study including staff-perceived factors and facility/programrelated factors are concepts to be explored for the purposes of examining their influence on treatment allocation. Information related to staff demographics (gender and race), years of experience in agency, facility and juvenile corrections as well as position and facility assignment are included. Variables included in the examination of facility/program-related factors relate to agency and facility descriptions of working environments. These variables include items such as average daily population, cost per day of youth, bed capacity and mean age of staff. Individual facility environments are described using the following variables: bed capacity, gender and race among staff, mean age, number of full time employees, and number of staff identified with disabilities. 
Additionally, variables used to describe the overall mental health status and treatment provision of the study sample were included. Mental health status is defined by presence of a diagnosis outlined in the Diagnostic and Statistical Manual Fourth Edition Text Revision (DSM-IV-TR). IQ score and number of traumatic experiences reported on the MAYSI assist in illuminating youth demographics. Treatment variables include presence of previous mental health treatment and treatment need identified at intake. Finally, a variety of treatment variables are included to demonstrate the various approaches to mental health treatment found in the study. These were provided to further illustrate the convolution of youth in OYA and to provide significant context for the qualitative findings.

Next, the methodology section is offered to describe the mixed-method design chosen for this study. Quantitative and qualitative methods are presented together in order of question design. For example, quantitative methods will be discussed first as the first research question employs quantitative methods. Then, additions for qualitative methods will be provided where appropriate. 


\section{Chapter Three: Methodology}

\section{Design}

This study's overall objective is to determine which factors influence treatment allocation among youth in closed custody facilities in the OYA. To achieve this, a mixed methods design was used. Due to the relatively unknown aspect of treatment resource allocation in closed custody settings, a mixed method design allows for a broader understanding of the research problem to be investigated. The lived experiences of OYA staff will further inform researchers of quantitative investigations of the need to investigate additional factors such as organizational factors as they may influence decision-making. Using strictly quantitative data in this study would limit the analysis of the investigation. Using the logic of a mixed methods approach, neither quantitative nor qualitative methods are sufficient to capture the details of the situation presented in this research problem to be explored in OYA. Both quantitative and qualitative data offer a more inclusive analysis, when used together, and they complement each other (Creswell, Fetters, and Ivankova, 2004). The unit of analysis for quantitative methods will be individual youth cases, while the focus will explore patterns across multiple cases and will not discuss individual cases. Both approaches will be described in the following sections with the quantitative approach discussed first.

Research questions two and three were explored using qualitative methods. Focus group methodology was used to explore the second research question. A series of four focus groups including an average of seven participants per group were facilitated. This researcher facilitated all focus groups independently. An OYA contracted employee took 
hand written notes for record keeping purposes. Focus groups were explored the experiences of OYA staff and their observation of who is afforded treatment and what factors influence allocation. Discussion guided by the facilitator explored factors influencing the decision to allocate treatment youth in custody. Focus group methodology was chosen for its ability to seek a sizable amount a data on a very specific topic of interest. In comparison to individual participant interviews, they serve as an efficient way at gaining equivalent amounts of data. Morgan (1997) also highlights the usefulness of focus groups due to the reliance on interaction in the group to generate data. Insightful observations and observations discussed in the focus group can reveal rich substantive data to the researcher. The following paragraphs give a brief summary of the proposed population and sample parameters.

\section{Participants}

Agency. The OYA is a large public juvenile justice system serving over 2,000 youth and 36 counties in the state of Oregon. The mission of the Oregon Youth Authority is "to protect the public by holding youth offenders accountable and providing for reformation in safe environments," (OYA at a Glance, 2011, p.1). The agency serves the state's delinquent youth from age 12 to 24 . The majority of youth in close custody are between the ages of eighteen and twenty. Demographically, OYA serves about 54\% Caucasian youth, followed by $24 \%$ percent Hispanic youth, and 16\% percent African American youth (OYA Quick Facts, 2017). Among youth in the OYA, $88 \%$ of females and $75 \%$ of males are diagnosed with a mental health disorder, excluding Conduct 
Disorder. Additionally, $73 \%$ of females and $63 \%$ of males are identified with a substance abuse or dependence (OYA Biopsychosocial Summary, 2016).

OYA represents two primary values for the state of Oregon, to ensure public safety and to provide opportunities for reformation. According to OYA, public safety is achieved through the use of sanctions and interventions to reduce offenders' risk factors for reoffending (OYA at a Glance, 2011). Reformation is accomplished through structured case planning processes using risk assessments and effective interventions. During the 2009-2011 legislative sessions, funding was approved for the agency at 312.1 million dollars (total funds) of which general funds included 266 million dollars. Just over half (51\%) of the agency's budget is spent in facilities. The OYA employs approximately 1,135 staff, which includes 153 employees serving in management positions. As a whole, about $38 \%$ of the staff is female, slightly more than two percent of staff has disabilities, and seventeen percent are persons of color (OYA at a Glance, 2011).

The OYA provides an excellent location for a study exploring allocation of treatment resources to youth in care. Given its large size, dual value mission, and diversity of staff characteristics, it is a viable case to investigate and represent the JJS on a small scale. In order to assess the prevalence of mental health disorders in the agency, the OYA utilizes GAP (no series of words for extended tile exist) surveys to identify youth in custody with a mental health diagnosis. GAP surveys designed by the OYA have been conducted every other year since 2000. On April 1 of the survey year, data are collected on all youth in OYA custody (OYA Mental Health GAP Survey, 2008). All 
youth are included in the survey that resides in closed custody facilities. GAP data is collected with both a JJIS file review and a clinical file review. GAP surveys are used by the agency to identify gaps in service delivery as well as advocate for services during the budget process. Items such as: "Does the youth have an Axis 1 diagnosis, excluding conduct disorder?" and "Does the youth have one or more suicide attempts in past 3 years?" are included in the GAP survey. The 2008 GAP survey indicated $70 \%$ of youth incarcerated in OYA facilities have a mental health diagnosis (excluding Conduct Disorder; including anxiety, mood, psychotic, and behavioral disorders of childhood onset). Prevalence rates in OYA mirror rates found in existing national studies. With such a large offender population identified with a mental health disorder, more evidence is needed to determine which youth are receiving services and which factors determine how resources are allocated. A total of nine individual OYA facilities were represented in the study.

Youth and staff. OYA has a population of approximately 900 youth residing in closed custody. This census can fluctuate given the time of year and may vary. To determine a sufficient sample size for quantitative methods in this study, a two-year parameter was used to identify youth for the sample. All youth committed to a youth correctional facility during January 1, 2007 and December 31, 2009 were included in the sample. Given the possibility that census can fluctuate within the OYA; the following determination of a minimum sample size was initially determined during the design of this study. This yielded a sample of 594 youths $(\mathrm{N}=594)$. Among the 504 cases, 294 are male and 300 are female. Male and female populations were assessed independently. 
Participant selection for focus groups included a purposive sampling method. OYA staff working in closed custody living units working youth offenders was recruited to participate in focus groups. Through the use of electronic communication and an agency email to all facility staff, requests for participation were solicited. This yielded a total sample of 28 staff $(\mathrm{N}=28)$. Among those staff, 22 were male and 6 were female. Non-direct care staff was excluded from the focus group recruitment. This resulted in a random sample from the remaining larger group of OYA staff who meet criteria to be included. This type of group composition will allow for free-flowing conversations among participant focus group site locations will be conference room type location at each facility. Since multiple staff within OYA likely experiences the research topic investigated, attempts to achieve segmentation for focus group composition were imposed. Segmentation allows for some homogeneity in the participants and provides for common experiences to emerge. This technique also provides an opportunity to examine differences between the focus group as they relate to the topic of investigation (Morgan, 1997). However, due to scheduling constraints, this was not achieved. Each participant was enumerated given $\$ 15$ gift card for his or her participation in the focus group.

\section{Measures}

For the purposes of this study mental health treatment is defined by treatment including one of the following mental health treatment resources: Cognitive Behavioral Therapy, Dialectical Behavioral Therapy, Seeking Safety or other treatment targeting trauma, individual psychotherapy, and psychiatric services. Presence or absence of mental health treatment recommendations was measured using a dichotomous response 
(yes or no). Demographic information on youth was measured using available existing data, which includes youth history and identification data in JJIS. Behavioral incidents for youth while in custody were measured using the YIRs in JJIS. Recorded mental health status will be measured using existing psychological and psychiatric records. Treatment allocation was measured using the JJIS intervention listings, JJIS contact treatment group notes and other various narrative descriptions archived in facility hard files.

Every youth who enters OYA receives a number of assessments determined by the following phases (N. Reed, personal communication, April 26, 2011). The first phase includes an initial mental status assessment conducted by a master's level mental health professional, an administered MAYSI-2 instrument during the initial two hours of arrival to a closed custody facility. The second phase includes an initial intake psychological evaluation consisting of the Structured Clinical Interview for the Diagnostic and Statistical Manual, Fourth Edition-TR (SCID), the Symptom Checklist 90-R and the Inventory of Suicide Oreintation-30. A brief review of the MAYSI and the SCID are provided in the following paragraphs. Youth are re-assessed at subsequent points in their stay with OYA only if there is indication for further testing in areas such as treatment planning, intelligence testing, and to corroborate behavioral observations after an extended period of observation at the facility (N. Reed, personal communication, April 26, 2011). This information may be limited on all individuals in the sample since not every youth may receive subsequent assessments. The MAYSI was developed in 1998 to screen symptoms of mental and emotional distress in youth entering the JJS between the 
ages of 12-17 years of age (Archer, Bisbee, Spiegel, Handel, and Elkins, 2010).

According to Grisso and Quinlan (2005) approximately 32 states use the MAYSI in their JJS. The MAYSI is a 52 item instrument asking raters to answer "yes" or "no" to each item as experienced "within the past few months." The instrument consists of seven scales including: Alcohol/Drug use, Angry-irritable, Depressed/Anxious, Somatic Complaints, Suicide Ideations, Traumatic Experiences and Thought Disturbances. Scoring is based on a continuum of "yes" responses to scale questions. Scales are grouped into "cut-offs" and result in a score identified to be in the caution range or warning range. Respondents who score in the warning range are likely to have "clinically significant" needs (Archer et al, 2010). Grisso and Barnum (2006) report the internal consistency of MAYSI domains with the exception of the Traumatic Experiences domain to include alpha coefficients from .61 to .86 .

A licensed psychologist utilizing a battery of common tests conducts the intake psychological evaluation. At the minimum, this sequence includes a SCID containing the following: the use of required questions; operational criteria from the DSM, a categorical system for rating symptoms, and a set of rules for arriving at a final diagnosis. The SCID instructions also guide the interview to establish review of alternative sources of information regarding the subject of the interview. Reliability is found to be good to excellent level of diagnostic reliability (Ventura, Lieberman, Greene, Shiner, and Mints, 1998). The Symptom Checklist 90-R (SCL-90), and Inventory of Suicide Orientations-30 are also commonly used tests in psychological assessments. The SCL-90-R is a brief inventory to assess psychological symptoms across eight dimensions of symptom 
categories. It has been found to have good test-re-test and internal consistency reliability as well as construct and convergent validity (Derogtis and Melisaratos, 1983). The Inventory of Suicide Orientations-30 demonstrates good reliability and strong convergent validity. The Inventory of Suicide Orientations-30 measures five dimensions connected to suicide. They are as follows: suicide ideations, hopelessness, and inability to cope with emotions, low self-esteem, social isolation and withdrawal (Osman et al., 2005). To collect the necessary qualitative data, a short survey will be given to participating focus group staff in order to measure race, gender, staff position, and location of facility assigned. The focus group questions will serve as the measure for the independent variables, including staff perceptions of factors influencing allocation of mental health treatment resources.

\section{Procedures}

Youth facility files were reviewed for recorded information pertaining to their care and custody while in facilities. Hard files housed in facility medical clinics and state archives were reviewed for recorded mental health evaluations and other pertinent clinical information for OYA youth. Additional data collection included a review of various electronic youth files. Primarily, the JJIS system was used to extract data on youth information and treatment programming for not located in hard files. Clinical files were reviewed for information relating to mental health diagnosis, MAYSI scores and recommendations for treatment. Diagnosis, MAYSI scores and recommendations for treatment were taken from direct psychological evaluations that are maintained in hard copy form in the clinical file. Some Psychological evaluations were found in JJIS, while 
others were located in clinic hard files. A clinical diagnosis and youth checklist instrument was used for recording data extracted from hard files. This instrument can be found in Appendix A. JJIS was used to identify referrals and interventions youth offenders were assigned. JJIS was used to identify which facilities and treatment programs were assigned to youth offenders. Multi-disciplinary team (MDT) recommendations were reviewed through JJIS documents to identify recommendations for how treatment should be allocated. These documents include offender contact notes written by qualified mental health professionals and other staff, as they provide important information regarding recommendations and referrals. Recordings from the focus groups were transcribed to allow for further analysis. Data collected from focus groups was analyzed using thematic analysis. A SPSS Codebook was designed to assist in the recording and organizing of the extracted data. JJIS identification numbers were used rather than participant names for confidentiality. SPSS (PASW 19.0) statistical software was used in the analysis of the data collected for this study.

\section{Data Analysis}

A cross-sectional data analysis was conducted on the available quantitative data. Descriptive statistics were used to describe the sample. Means and standard deviations were reported for continuous variables while frequencies and percentages were used to analyze categorical variables. Youth, staff and facility level quantitative data are were analyzed using a similar analysis. Although, in the initial design of the study a logistic regression was proposed, due to data availability limitations, alternative analyses were implemented to preserve the opportunity to present the data. A logistic regression 
analysis was initially identified as the most effective way of assessing likelihood of a youth offender receiving mental health treatment. This analysis could have determined the likelihood of this event associated with independent variables (commitment offense, various demographics, and YIRs). Due to the issues with data accessibility, Odds ratios were not used to report the increase or (decrease) in odds of receiving mental health treatment when considering influencing factors like race and offense type. Instead, data is analyzed using a more conservative statistical analysis.

To answer the second research question of what factors does staff perceive as influencing the decision to allocate mental health treatment to youth offenders? Focus group data was analyzed using thematic analysis. Thematic analysis is the chosen method for analysis due to its ability to assist the researcher in gaining insight into a phenomenon (Boyatzis, 1998). Thematic analysis serves as a way to enhance and clarify the quantitative results. Furthermore, the use of thematic analysis will aid this researcher's ability to expand dissemination of the results to the targeted audience of OYA administrators and stakeholders. An inductive, data-driven approach was used to identify codes to establish themes in the raw data collected from focus groups. The unit of data analysis was each separate focus group of OYA staff. The following process was used to identify codes for each theme as recommended by Boyatzis (1998): A label name was chosen (e.g. perception of training); A definition of what the theme concerns was devised; A description of how to know when the theme occurs was defined (e.g. the respondent expresses experiences with training relating to type, frequency, assignment to, availability, cost or quality); A description of any qualifications or exclusions to the 
identification of the theme was selected. Criterion-referenced material was used to identify similarities and differences in staff perception of factors influencing the allocation of treatment resources to youth offenders. The unit of coding was staff perceptions of factors influencing allocation of resources to youth offenders. This illuminated themes in social constructions of youth offenders and its impact on resource allocation. Finally, as part of the analysis, the steps suggested by Boyatzis (1998) were provided as a guide. First, raw information was reduced using an outline. Second, themes within focus groups were identified. Third, themes found within each focus group were compared to one another for preliminary themes. Fourth, themes and codes were described in terms of the tenets of social construction theory. Since data from focus groups are unique to OYA staff, a brief description of the units of analysis with accompanying themes proved to be sufficient (Boyatzis, 1998). Since this study used a small sample for focus groups and is limited to the OYA, this method of sharing collected observations is highly desired. As the advantages of this method are described earlier in this section, a limitation of this analysis is the limited number of raters available to code raw data to test for reliability. For example, a double coding technique to check for reliability as discussed in Boyatzis (1998) were not be available due to the limited scope and time of this study. This proved to be a limitation. There were only two coders. Each coder was provided with a label and definition guide, who included labels, definitions for each label, exclusions and specific examples. Each coder independently reviewed and coded responses using identification techniques of color highlighting. Each similarly highlighted statement was then individually placed onto a large sheet of poster paper. 
Each segment of color-coded statements fitting the label guidelines emerged into salient themes. From there, a review and interpretation of the themes assisted in answering the third research question of do the quantitative results and the qualitative findings about factors influencing the allocation of mental health treatment converge? This was completed through reviewing each factor area (youth, staff and facility/program) and determining which quantitative data provided a framework for which focus group themes could be better understood. Next, results from this study will be presented. 


\section{Chapter Four: Results}

Results from both quantitative and qualitative approaches will be presented using the same framework outlined in the literature review. First, results of the youth related data are discussed. The first research question was not answered in this study. There were significant issues with data sources to include multiple locations of clinic and facility files, difficulty ascertaining whether data was duplicated or recorded exclusively in one data source, and geographic location of files. The wide variability of types of treatment, names of treatment and types of recommendations will further be discussed in the limitations portion of this study. Due to these complexities, assuring the ability to secure all data for each quantitative variable is this study was limited. Additionally, the amount of time to extract data from various data sources proved to be daunting and required additional resources beyond the scope of this study. Given these limitations with access to data, youth data will be presented in a similar spirit, recognizing the importance of individual characteristics and their potential influence on receiving treatment. Second, themes that emerged through thematic analysis of the four focus groups will be highlighted. Third, themes and relevant parallel findings from the youth, staff and facility data will be offered to illustrate important contextual factors.

\section{Youth}

Among the youth included in the sample, $48.7 \%(\mathrm{n}=294)$ were male and $49.7 \%$ $(\mathrm{n}=300)$ were female. The majority of youth were White $(59.6 \%, n=360)$, followed by smaller proportions of Hispanic youth $(19.7 \%, n=119)$ and African American youth $(12.6 \%, n=76)$. Table 1 illustrates the various characteristics associated with youth, 
including demographics, offense related information, behavioral indicators and treatment information. A combination of the remaining 36 counties made up the highest number commitments $32.6 \%, \mathrm{n}=197)$. Overwhelmingly, the single county of commitment for the majority of youth was Multnomah County $(19.7 \%, n=119)$. Second was Marion County with about $9.8 \%$ ( $n=59)$ of youth committed to OYA. Commitment to OYA was the most common disposition type with just over $46 \%(n=460)$ of the youth mandated in juvenile court. Among Department of Corrections (DOC) youth in the custody of the OYA, 14.4\% $(\mathrm{n}=87)$ were waived, while 5.6\% (34) has Measure 11 sentences. The preponderance of offenses included assault $(26.7 \%, \mathrm{n}=161)$, sex offenses $(19.9 \%, \mathrm{n}=120)$, and theft $(12.7 \%$, $\mathrm{n}=77)$.

Behavioral indicators, mental health status and treatment information were found in a smaller subsample of youth. Table 1 further specifies each subsample with number of youth included in each. Within the subsample for behavioral indicators, there is a gap in number of incident reports are various time points in custody. The highest prevalence of incident reports $(M=26.1, S D=30.4)$ and incidents of isolation $(M=26.1, S D=41.8)$ occurred at the 36-month mark respectively. Within the subsample of youth who had a suicide attempt within the last three years, $8.4 \%(\mathrm{n}=51)$ had one attempt and $3.6 \%(\mathrm{n}=22)$ had multiple attempts. Mental health status is represented by a large mass of youth with diagnoses in clinical categories of mood disorders $(28.1 \%, \mathrm{n}=167)$, anxiety $(25.3 \%$, $\mathrm{n}=150)$, substance use $(27.4 \%, \mathrm{n}=163)$ and dependence $(28.6 \%, \mathrm{n}=170)$. Additionally, a large number of youth had IQ scores over $80(26.8 \%, n=162)$ and $11.6 \%(n=70)$ of youth reported experiencing at least one traumatic experience. Among those youth with more 
than one traumatic experience, $11.6 \%(n=70)$ reported four. Finally, in regards to proportion of youth with previous treatment, $41.6 \%(\mathrm{n}=251)$ participated in inpatient treatment prior to commitment, while $89 \%(n=14.7)$ participated in outpatient treatment. At intake, a large margin of youth were identified as having a mental health treatment need $(63.6 \%, n=384)$.

For those who were identified as receiving various treatment types, a large section of youth received cognitive behavioral treatment $(18 \%, n=109)$. Dialectical Behavior Therapy was the second highest category of prescriptive treatment received $(8.6 \%, \mathrm{n}=52)$. Youth also received additional types of treatment including treatment aimed at reducing suicide risk and psychiatric services. Youth received treatment for suicide risk at various time intervals. The majority of youth had frequency of contact other than weekly, monthly or quarterly $(4.1 \%, n=25)$. Similarly, $37.2 \%(n=226)$ of youth received follow up services with psychiatry (after initial appointment) in a variety of weekly intervals other than one to four weeks. Among the segment of youth receiving medication management services with psychiatry, most of them were seen on a monthly basis $(8.3 \%, \mathrm{n}=50)$.

Table 1

Descriptive Statistics for Participants

\begin{tabular}{|c|c|c|c|}
\hline \multirow{2}{*}{\multicolumn{4}{|c|}{$\begin{array}{l}\text { Variable } \\
\text { Youth characteristics }(\mathrm{N}=549)\end{array}$}} \\
\hline & & & \\
\hline Male & 294 & 48.7 & \\
\hline Female & 300 & 49.7 & \\
\hline Age & & & $21.1(2.3)$ \\
\hline Race & & & \\
\hline African American & 76 & 12.6 & \\
\hline Asian & 11 & 1.8 & \\
\hline Hispanic & 119 & 19.7 & \\
\hline Native American & 25 & 4.1 & \\
\hline White & 360 & 59.6 & \\
\hline
\end{tabular}


Other/Unknown

County of Commitment

Multnomah

Washington

Clackamas

Lane

Marion

Jackson

Deschutes

Linn

All others

Disposition

Commitment to

OYA

DOC-M11

DOC-Other

DOC-Reduced

DOC-Waived

Offense Type

Arson

Assault

Burglary

Criminal mischief

Criminal other

Criminal trespass

Disorderly conduct

Harassment

Homicide related

Person other

Robbery

Sex offense

Substance/alcohol

Theft

Weapons
3

.5

$119 \quad 19.7$

$45 \quad 7.5$

$58 \quad 9.6$

$\begin{array}{ll}57 & 9.4\end{array}$

$\begin{array}{ll}59 & 9.8\end{array}$

$35 \quad 5.8$

$10 \quad 1.7$

$14 \quad 2.3$

$197 \quad 32.6$

$460 \quad 76.2$

$34 \quad 5.6$

$1 \quad .2$

$12 \quad 2.0$

$87 \quad 14.4$

$12 \quad 2.0$

$161 \quad 26.7$

$\begin{array}{ll}161 & 8.4\end{array}$

$18 \quad 3.0$

$33 \quad 5.5$

2.3

12.2

$\begin{array}{ll}4 & .7\end{array}$

$9 \quad 1.5$

$10 \quad 1.7$

$\begin{array}{ll}41 & 6.8\end{array}$

$120 \quad 19.9$

$45 \quad 7.5$

$\begin{array}{ll}77 & 12.7\end{array}$

$\begin{array}{ll}10 & 1.7\end{array}$

Behavior

6 months $(\mathrm{n}=60)$

12 months $(\mathrm{n}=55)$

24 months $(\mathrm{n}=49)$

36 months $(\mathrm{n}=42)$

Incidents of isolation

6 months $(\mathrm{n}=60)$

12 months $(\mathrm{n}=55)$

24 months $(\mathrm{n}=49)$

$5.9(7.1)$

$10.5(12.6)$

$19.5(22.6)$

26.1(30.4)

36 months $(\mathrm{n}=42)$

$6.8(10.7)$

$11.7(17.5)$

21.2(32.8)

26.1(41.8)

Suicide Attempt (within last

3 years $)(n=468)$

No attemp

395

65.4

1 attempt

51

8.4

Multiple attempts

22

3.6 
IQ Score $(n=211)$

Over 80

162

43

70-80

Below 70

6

26.8

7.1

Number of traumatic

experiences $(\mathrm{n}=341)$

$\begin{array}{lcc}\text { None } & 53 & 8.8 \\ \text { One } & 70 & 11.6 \\ \text { Two } & 62 & 10.3 \\ \text { Three } & 49 & 8.1 \\ \text { Four } & 70 & 11.6 \\ \text { Five } & 37 & 6.1\end{array}$

Previous treatment (prior to

commitment $)(n=354)$

Inpatient

251

41.6

Outpatient

89

14.7

Other

14

2.3

Treatment need identified at intake $(n=414)$

Yes

63.6

No

30

5.0

Initial diagnoses $(\mathrm{N}=594)^{* *}$

Anxiety

150

25.3

Impulse related

94

15.8

Mood disorder

167

28.1

Psychotic related

8

1.3

SUBS abuse

163

27.4

SUBS dependence

170

28.6

Personality

63

10.6

Other

86

14.5

None

196

33.0

Type of treatment received** $(\mathrm{n}=411)$

Trauma based

Indiv. counseling

DBT

CBT

Suicide risk specific $(\mathrm{n}=82)$

At least weekly

At least monthly

At least quarterly

Other

None

Psychiatric follow up (First

9

1.5

40

6.6

52

8.6

109

18.0

12

2.0

11

16

1.8

$25 \quad 4.1$

2.6

$18 \quad 3.0$

subsequent appointment)

$(\mathrm{N}=594)$

In 1 week

51

8.4

In 2 weeks

156

25.8

In 3 weeks

14

2.3 


\begin{tabular}{|c|c|c|c|c|}
\hline & In 4 weeks & 37 & 6.1 & \\
\hline & Other & 226 & 37.4 & \\
\hline & None & 110 & 18.2 & \\
\hline \multicolumn{5}{|c|}{$\begin{array}{l}\text { Medication management } \\
\text { (overall) }\end{array}$} \\
\hline & At least weekly & 3 & .5 & \\
\hline & At least monthly & 50 & 8.3 & \\
\hline & At least quarterly & 5 & .8 & \\
\hline & Other & 5 & .8 & \\
\hline & None & 531 & 87.9 & \\
\hline \multicolumn{5}{|c|}{ *Staff characteristics $(\mathrm{N}=28)$} \\
\hline \multicolumn{5}{|c|}{ Gender } \\
\hline & Male & 22 & 78.6 & \\
\hline & Female & 6 & 21.4 & \\
\hline Age & & & & $47.3(9.9)$ \\
\hline \multicolumn{5}{|c|}{ Years of experience } \\
\hline & OYA & & & $9.4(6.7)$ \\
\hline & Facility & & & $8.1(6.2)$ \\
\hline & Juvenile corrections & & & $11.4(7.2)$ \\
\hline \multicolumn{5}{|c|}{ 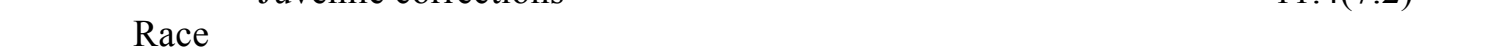 } \\
\hline & African American & 3 & 10.7 & \\
\hline & Hispanic & 1 & 3.6 & \\
\hline & Native American & 1 & 3.6 & \\
\hline & White & 23 & 82.1 & \\
\hline \multicolumn{5}{|c|}{ Position } \\
\hline & GLC & 8 & 28.6 & \\
\hline & $\mathrm{TM}$ & 2 & 7.1 & \\
\hline & YCUC & 6 & 21.4 & \\
\hline & QMHP & 7 & 25.9 & \\
\hline & $\mathrm{PD}$ & 2 & 7.1 & \\
\hline & OMS & 1 & 3.6 & \\
\hline & Other & 2 & 7.1 & \\
\hline \multicolumn{5}{|c|}{ Facility } \\
\hline & MacLaren & 14 & 50.0 & \\
\hline & Hillcrest & 5 & 17.9 & \\
\hline & Rogue Valley & 2 & 7.1 & \\
\hline & North Coast & 2 & 7.1 & \\
\hline & Eastern & 1 & 3.6 & \\
\hline & Riverbend & 2 & 7.1 & \\
\hline & Oak Creek & 2 & 7.1 & \\
\hline \multicolumn{5}{|c|}{$\begin{array}{l}\text { Note. } * \text { Total percentages are not } 100 \text { because of rounding. **Total frequencies exceed } 594 \text { due to } \\
\text { youth with more than one diagnosis. CBT=Cognitive Behavioral Treatment; DBT= Dialectical } \\
\text { Behavior Therapy; GLC=Group Life Coordinator; OMS=Office of Minority Services; } \\
\mathrm{PD}=\text { Program Director; QMHP=Qualified Mental Health Professional; SUBS=Substance; } \\
\text { TM=Treatment Manager; YCUC=Youth Corrections Unit Coordinator. Number of traumatic } \\
\text { experiences reported on MAYSI screen. Behavior and isolation are reported as frequencies at } \\
\text { respective time intervals. }\end{array}$} \\
\hline
\end{tabular}




\section{Staff}

Table 1 also displays demographic information of the 28 focus group participants. The majority of participants were male $(78.6 \%, n=22)$ and 6 were female $(21.4 \%)$. The average age of participants was 47.3 years $(\mathrm{SD}=9.9)$ and had just over a decade of work experience in juvenile corrections $(\mathrm{M}=11.4, \mathrm{SD}=7.2)$. Mean years of experience in OYA and at their assigned facility was $9.4(\mathrm{SD}=6.7)$ and $8.1(\mathrm{SD}=6.2)$ respectively. Most participants were White $(82.1 \%, n=23)$ and $50 \%(n=14)$ of the participants were representing MacLaren, the largest facility in the agency.

Among Likert scale responses, most staff felt they had a say in whether or not a youth receives treatment. $36 \%$ of staff $(n=10)$ strongly agreed with this sentiment. Additionally, the majority of staff reported they did have an opportunity to participate in the decision making process to allocate treatment to youth $(32 \%, n=9)$. Staff answered in a variety of ways when asked about the power and influence of a parole officer (PO) in the decision making process to allocate treatment. Table 2 displays the various opinions among staff in regards to PO influence. $42 \%(n=12)$ of staff agreed the role of a PO has the most amount of influence in multi-disciplinary team decision-making.

Overwhelmingly, staff felt youth should be involved in the decision making process $(68 \%, n=19)$. Finally, 18 staff $(65 \%)$ agreed that decisions regarding treatment were made without the involvement of others in a youth's case (parents, teachers, etc.).

\section{Table 2}

Frequency of Participant Responses Using Likert Scale Measurement

Question

"Do you feel you have a say in

whether or not a youth receives

treatment?" (Strength of 
$\begin{array}{lll}\text { Strongly disagree } & 5 & 18\end{array}$

Slightly disagree $\quad 1 \quad 4$

Neutral $3 \quad 11$

$\begin{array}{lll}\text { Slightly agree } & 7 & 25\end{array}$

Strongly agree $\quad 10 \quad 36$

No response $\quad 2 \quad 4$

"Do you feel you have enough opportunity to voice your input in the decision making process?

(Strength of Agreement)

$\begin{array}{lll}\text { Strongly disagree } & 6 & 21\end{array}$

$\begin{array}{lll}\text { Slightly disagree } & 3 & 11\end{array}$

Neutral $\quad 3 \quad 11$

$\begin{array}{lll}\text { Slightly agree } & 6 & 21\end{array}$

Strongly agree $\quad 9 \quad 32$

No response

14

"In your perception, do you

believe a youth's parole officer

has the most amount of power

and influence in the decision to

allocate treatment to youth?

(Strength of Agreement)

$\begin{array}{lll}\text { Strongly disagree } & 2 & 7\end{array}$

$\begin{array}{lll}\text { Slightly disagree } & 6 & 21\end{array}$

$\begin{array}{lll}\text { Neutral } & 6 & 21\end{array}$

$\begin{array}{lll}\text { Slightly agree } & 6 & 21\end{array}$

$\begin{array}{lll}\text { Strongly agree } & 6 & 21\end{array}$

No response

7

"Do you agree that youth should be included in the

decision making process?"

(Strength of Agreement)

Strongly disagree $\quad 0 \quad 0$

$\begin{array}{lll}\text { Slightly disagree } & 0 & 0\end{array}$

Neutral 0

$\begin{array}{lll}\text { Slightly agree } & 8 & 29\end{array}$

$\begin{array}{lll}\text { Strongly agree } & 19 & 68\end{array}$

No response 114

"Do you agree decisions are

made without input from others

involved in a youth's case?

(Strength of Agreement)

Strongly disagree

3

11

Slightly disagree

Neutral

$3 \quad 11$

Slightly agree

2 7

Strongly agree 
No response

2

7

Note. $\mathrm{N}=28$. Total of percentages is not 100 because of rounding.

Qualitative thematic analysis yielded three particular themes. Each theme is presented with a short description of each and relevant participant (PAR) responses that were meaningful and significant. All themes were constructed based on perceptions of participants as elements in which they believe influence the identification of youth for treatment. A few examples of narrative statements found in clinic and facility files mentioned by a contracted psychologist of a smaller facility are offered with the first theme. Since focus group participants included both clinical and non-clinical staff, these examples serve are significant in understanding the discourse used to describe treatment need. These narrative statements emphasis the power and influence clinical staff have on the allocation of treatment.

The ways in which staff is exposed to mental health knowledge is important.

For the purposes of interpreting the themes presented in this section, knowledge is defined as an awareness or familiarity often gained by experience or situations in which staff is exposed. Participants spoke regularly about the methods in which they were exposed to knowledge of mental health symptoms and treatment programs in the OYA. Often, participants mentioned they received a substantial amount of "on the job training." They further explained training was provided more formally through the agency's training academy at the onset of their employment. Participants mentioned most of their experience and knowledge regarding mental health was from previous employment in juvenile corrections, residential treatment or other work with youth and children. 
FG3Participant A offered this description of how he learned of mental health treatment in the agency: "Previous community work. Hiring process emphasized mental health treatment at MacLaren." FG1Participant A added: "on the job training and over the years meeting the needs of youth, moving to more a mental health focus." Most participants reported being exposed to knowledge through interactions while working within the agency. Of significance are the following narratives reported in one youth's file. An agency-contracted psychologist employed at a smaller facility routinely administered psychological evaluations that were subsequently read by staff in treatment decision making roles. The following statements were observed: "unfortunately, this youth is probably too damaged at this point for this (evaluation/treatment) to have any impact." In additional files, the following statement was observed in reference to which youth and what types of treatment should be offered: "the standard armamentarium of treatment related for an externalized, conduct disordered youth would apply." Moreover, participants were clear on how youth are viewed and with which lens often influences whether or not a youth is allocated treatment. Factors such as behavior, age, commitment offense and mental health symptoms were also discussed. FG4 Participant T reported "younger youth get more help....there's a perception older youth have it figured out." Observation of mental symptoms during development was an important distinction in the discourse. FG1 Participant D clarified "younger youth are less able to hide symptoms...they receive more treatment. Older youth are better able to mask symptoms and go untreated." 


\section{The ways in which agency training on mental health and treatment is}

provided is important. Participants addressed concerns that formal training on mental health and treatment is rare. They went on to clarify informal training, which is provided, is often inconsistent. Furthermore, they felt the agency has strict definitions of what training are provided and this is a significant barrier. FG1Respondent B mentioned, "agency training is not adequate. You have to seek out your own training through other sources.” The need for training for non-mental health staff was emphasized. FG4 Participant Y stated staff "need more mental health training for GLCs, so (we do not just pass off to the Q." Other barriers staff identified to receiving training included systematic issues, funding, time off to attend, and an agency belief that training will be provided informally by QMHPs. Staff also discussed an ancillary issue to agency training. Staff believes attitudes among staff influence the utilization of mental health training as staff makes efforts to support youth. FG1 Participant D mentioned, "staff see mental health as a weakness....there is a stigmatization of those seeking mental health treatment." Participants identified staff factors such as level of training or experience as well as level of investment as integral in the allocation of treatment.

\section{The ways in which staff experience organizational change influence}

treatment receipt. Significant facility/program factors such as the program culture, occurrence of change, leadership and beliefs of the program were discussed often by participants. Participants observed agency changes frequently. They believe change is driven by budget decisions. Further, they believe change directly impacts team cohesiveness and staffing resources. Facility/program factors are often impacted by 
priorities and change in the agency. FG1 Participant D stated, "treatment is more offense specific, but it provides general mental health services. There is a delineation between offense and mental health wraparound because OYA focused on offense specific treatment." Other facility/programs factors mentioned by participants were leadership, support, team meetings, and program beliefs. One significant statement alluded to a program's ability to support treatment. FG4 Participant U specified "strong leadership, staff expectations, and protected time" for treatment in programs is essential.

\section{Facility and Program}

Table 3 includes various agency and individual facility characteristics across eight years of funding and capacity. OYA had the highest allocated bed capacity during the 2005-2007 Legislative Annual Biennium (LAB). Cost per day (CDP) ranges from \$171 in 2005-2007 to \$231 in 2011-2013. Mean age of agency staff had little change over time. MacLaren and Hillcrest are the largest capacity facilities in the agency across all LABs. They also had the largest amount of full time staff (FTE). Small facilities' capacity sizes ranged from 25 to 75 beds during the four LABs. Primarily, across all facilities and LABs, the majority of staff was male. Mean ages of staff throughout all facilities and LABs were similar in range. A breakdown of each individual facility and information related to number of staff with disabilities is reported in Table 3. Race of all staff in each facility are also listed in Table 3 . The largest racial/ethnic category of staff spanning all LABs was White, followed by various additional diverse backgrounds. 
Table 3

Agency and Individual Facility Characteristics Across Legislative Bienniums

\begin{tabular}{|c|c|c|c|c|}
\hline Characteristic & $\begin{array}{c}\text { LAB } \\
2005- \\
2007 \\
(N=8) \\
M(S D) \\
f\end{array}$ & $\begin{array}{c}\text { LAB } \\
2007-2009 \\
(N=9) \\
M(S D) \\
f\end{array}$ & $\begin{array}{c}\text { LAB } \\
2009-2011 \\
(N=9) \\
M(S D) \\
f\end{array}$ & $\begin{array}{c}\text { LAB } 2011-2013 \\
(N=8) \\
M(S D) \\
f\end{array}$ \\
\hline \multicolumn{5}{|l|}{ Agency $(\mathrm{N}=1)$} \\
\hline $\begin{array}{l}\text { Average daily } \\
\text { population }\end{array}$ & 850 & 995 & 900 & 750 \\
\hline Cost per day & 171 & 196 & 219 & 231 \\
\hline Bed capacity & 345 & 295 & 295 & 270 \\
\hline Mean age & $\begin{array}{c}43.5(1 . \\
67)\end{array}$ & $42.6(3.0)$ & $43.7(2.9)$ & $44.2(2.0)$ \\
\hline \multicolumn{5}{|l|}{ Facility } \\
\hline \multicolumn{5}{|l|}{ Bed Capactiy } \\
\hline MacLaren & 345 & 295 & 295 & 270 \\
\hline Hillcrest & 180 & 180 & 180 & 180 \\
\hline Rogue Valley & 100 & 100 & 100 & 100 \\
\hline North Coast & 25 & 50 & 50 & 50 \\
\hline Eastern & 25 & 50 & 50 & 50 \\
\hline Riverbend & 50 & 50 & 50 & 50 \\
\hline Oak Creek & & 75 & 75 & 75 \\
\hline $\begin{array}{l}\text { Corvallis } \\
\text { House }\end{array}$ & 25 & 25 & 25 & \\
\hline Tillamook & 50 & 50 & 50 & 50 \\
\hline \multicolumn{5}{|l|}{ Male staff } \\
\hline MacLaren & 303 & 270 & 230 & 191 \\
\hline Hillcrest & 136 & 144 & 133 & 122 \\
\hline Rogue Valley & 85 & 87 & 81 & 73 \\
\hline North Coast & 31 & 38 & 36 & 36 \\
\hline Eastern & 31 & 34 & 31 & 31 \\
\hline Riverbend & 38 & 44 & 34 & 73 \\
\hline Oak Creek & & 28 & 35 & 40 \\
\hline Corvallis & 8 & 6 & 7 & \\
\hline House & & & & \\
\hline Tillamook & 30 & 29 & 26 & 25 \\
\hline \multicolumn{5}{|l|}{ Female staff } \\
\hline MacLaren & 120 & 109 & 77 & 54 \\
\hline Hillcrest & 103 & 101 & 74 & 57 \\
\hline Rogue Valley & 33 & 30 & 34 & 26 \\
\hline North Coast & 22 & 19 & 13 & 15 \\
\hline Eastern & 16 & 19 & 20 & 17 \\
\hline Riverbend & 16 & 9 & 11 & 8 \\
\hline Oak Creek & & 44 & 48 & 46 \\
\hline $\begin{array}{l}\text { Corvallis } \\
\text { House }\end{array}$ & 11 & 9 & 10 & \\
\hline $\begin{array}{l}\text { House } \\
\text { Tillamook }\end{array}$ & 22 & 18 & 20 & 15 \\
\hline \multicolumn{5}{|l|}{ Mean age } \\
\hline MacLaren & 43.1 & 43.1 & 43.6 & 45.0 \\
\hline
\end{tabular}




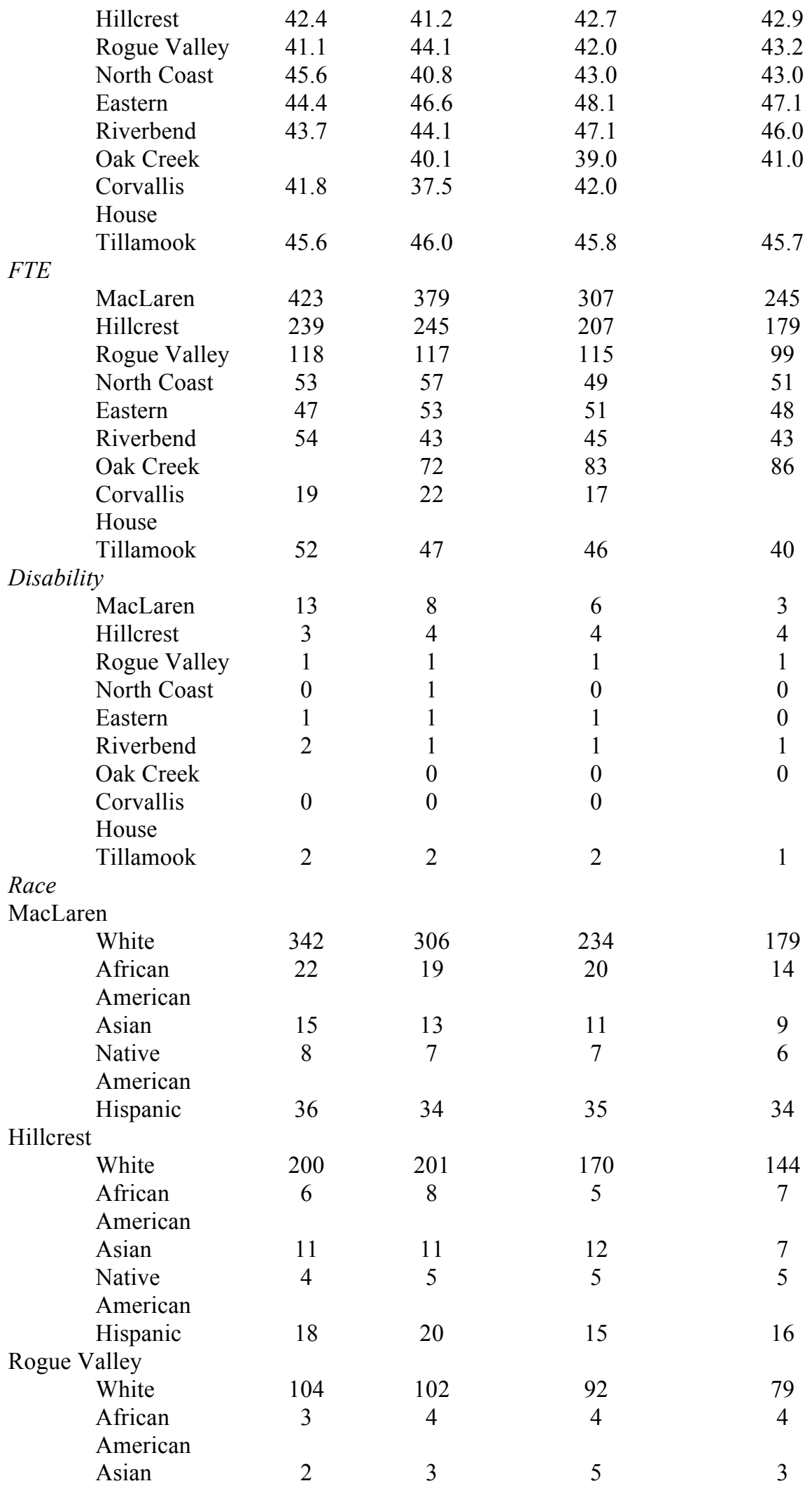




\begin{tabular}{|c|c|c|c|c|}
\hline Native & 1 & 0 & 2 & 1 \\
\hline American & & & & \\
\hline Hispanic & 8 & 8 & 12 & 8 \\
\hline \multicolumn{5}{|l|}{ North Coast } \\
\hline White & 47 & 52 & 44 & 44 \\
\hline African & 0 & 0 & 1 & 1 \\
\hline American & & & & \\
\hline Asian & 3 & 3 & 3 & 4 \\
\hline Native & 0 & 1 & 0 & 0 \\
\hline American & & & & \\
\hline Hispanic & 2 & 1 & 1 & 2 \\
\hline \multicolumn{5}{|l|}{ Eastern } \\
\hline White & 43 & 29 & 46 & 44 \\
\hline African & 0 & 0 & 0 & 0 \\
\hline American & & & & \\
\hline Asian & 1 & 1 & 1 & 1 \\
\hline Native & 2 & 3 & 3 & 2 \\
\hline American & & & & \\
\hline Hispanic & 1 & 2 & 1 & 1 \\
\hline \multicolumn{5}{|l|}{ Riverbend } \\
\hline White & 42 & 32 & 34 & 33 \\
\hline African & 0 & 4 & 7 & 8 \\
\hline American & & & & \\
\hline Asian & 0 & 0 & 0 & 0 \\
\hline Native & 1 & 1 & 1 & 1 \\
\hline American & & & & \\
\hline Hispanic & 3 & 3 & 3 & 1 \\
\hline \multicolumn{5}{|l|}{ Oak Creek } \\
\hline White & & 60 & 69 & 71 \\
\hline African & & 2 & 3 & 5 \\
\hline American & & & & \\
\hline Asian & & 4 & 5 & 4 \\
\hline Native & & 2 & 2 & 1 \\
\hline American & & & & \\
\hline Hispanic & & 4 & 4 & 5 \\
\hline \multicolumn{5}{|l|}{ Corvallis House } \\
\hline White & 17 & 19 & 15 & \\
\hline African & 0 & 0 & 0 & \\
\hline American & & & & \\
\hline Asian & 0 & 1 & 0 & \\
\hline Native & 0 & 0 & 0 & \\
\hline American & & & & \\
\hline Hispanic & 2 & 2 & 2 & \\
\hline \multicolumn{5}{|l|}{ Tillamook } \\
\hline White & 47 & 44 & 40 & 36 \\
\hline African & 0 & 0 & 2 & 2 \\
\hline American & & & & \\
\hline Asian & 3 & 1 & 3 & 2 \\
\hline Native & 0 & 0 & 0 & 0 \\
\hline American & & & & \\
\hline Hispanic & 2 & 1 & 1 & 2 \\
\hline
\end{tabular}


Note. $\mathrm{LAB}=$ Legislative annual budget. Cost per day reported in dollars. FTE $=$ Full time employees. Unit of analysis is individual facility. Facility closures during the 2005-2007 and 2011-2013 LAB included Oak Creek and Corvallis House respectively.

Agency and facility individualities strengthen the reported experiences and narrative descriptions of focus group participants. By thoroughly understanding the environments in which their observations are witnessed, a much more transformational level of understanding can be achieved. Interpretations and an overall dialogue of the study results will be presented in the following section. 


\section{Chapter Five: Discussion}

\section{Overview of Study}

The purpose of this study was to explore specific factors influencing treatment allocation among youth in OYA facilities. Primarily, the influence of youth level characteristics and agency/facility level characteristics on the allocation of treatment. This section will continue to highlight important framework and an overarching understanding of youth, staff and facility/program descriptions which give significant meaning to the qualitative elements of this study. The first research question is employed through quantitative methods. The second research question used qualitative methods through the use of focus groups. Focus groups were used to investigate staff perceptions about youth; program/facility and organizational factors that they perceived highly influenced the process of assigning treatment to youth offenders. Particular emphasis was placed on investigating staff perception and observations of factors at various levels of the agency. This illustrated particular nuances in the treatment provision decision-making process. Additionally, focus groups explored staff perception in regards to the decision making process when allocation of treatment resources is assigned to youth. The third research question is determining if the qualitative and quantitative results converge.

Focus group participant interactions highlighted consistent statements, thoughts, and beliefs about youth behavior, knowledge and training, agency leadership and organizational context. Furthermore, organizational themes illustrated staff insight about the presence of agency staffs' basic knowledge of mental health, availability of specific mental health trainings and how often staff experience change in the agency. Concepts 
introduced by staff included how aspects of prior mental health knowledge, previous work experience and leadership presence contribute to how youth are selected to receive treatment. Specifically, the organizational culture included a facility/program's approach to youth reformation as well as aspects of an effective team were identified as items that influence youth receiving treatment. In their study of effects of organizational climate and culture on access to mental health services for children in juvenile justice and child welfare systems, Glisson and Green (2006) found children who needed mental health services were much more likely to receive services when case managers belonged to more constructive work units, than those units with less constructive cultures. Their study of 588 children and 21 counties in Tennessee's juvenile justice system adds to the consideration of social context in the effectiveness of child welfare and juvenile justice systems. Specifically was it relates to the influence of organizational culture and service outcomes. It appears the finding support the work of Glisson and Green in similar aspects.

Data that emerged from focus group participant interactions included consistent statements, thoughts, and beliefs about youth behavior, knowledge and training, agency leadership and organizational context. The themes that result from the focus groups are appropriately interpreted as individual perceptions and experiences, through the lens of experienced correctional facility staff. Furthermore, organizational themes illustrated staff insight about the presence of agency staffs' basic knowledge of mental health, availability of specific mental health trainings and how often staff experience change in the agency. Focus group participants were keenly aware that multiple facets of the agency 
influence which youth are selected to receive treatment. Not only do the youth characteristics and behavior contributes, but also the previous mental health knowledge and work experience of staff alongside leadership presence. Taxman, Cropsey, Melnick and Perdoni (2008) explored organizations in the adult criminal justice system as part of a dataset from the National Criminal Justice Treatment Practices Survey (NCJTP). They examined various organizational factors influencing service delivery in organizations that provided co-occurring mental health and substance abuse services. Included in the list of factors measured were organizational demographics, climate and culture and leaderships style. Surprisingly, no significant differences were found among the level of services provided by various leadership styles. Given the unique nature and discourse of the JJS, it may be beneficial to replicate or examine similar factors in JJS agencies that provide similar services to youth. Differences in philosophy and mission between the adult and juvenile system may lend to varying leadership styles further effecting services. Another cultural issue in facilities is discussed in the work of Dvoskin and Spiers (as cited in Parker, 2009, p. 643). Due to aspects of culture within these communities, correctional officers may participate in the provision of mental health services to offenders. Often times, this may include verbal interaction in a therapeutic manner as well as offering consultation and observations of treatment issues with offenders such as medication side effects.

Finally, in studying organizational context and its influence on the use of current best practices in a juvenile correctional agency, Farrell, J., Young, D., and Taxman, F. (2011) uncovered staff who had more favorable views of their supervisor and were less 
cynical about their office's ability to change were more likely to use best practice supervision practices. Focus group participants spoke to the type of leadership and observable organizational change as factors influencing youth identified for treatment. Use of best practices in the field of mental health on identification, screening, assessment and referral are likely to be influenced by such context in OYA offices and facilities. This suggests the selection of agency managers and leadership should include an appraisal of qualities such as level of investment in treatment delivery, ability to support staff development and supervision and an aptitude to sustain the agency's philosophical approach despite frequent change. Assertion of the impact of differences in philosophy and approach towards offenders is further supported by the work of Oser, C., Knudsen, H., Staton-Tindall, M., Taxman, F., \& Leukefeld, C. (2009). Oser and colleagues claim "correctional institutions where the organizational culture is dominated by traditional criminal justice values may be less likely to invest in services that address the welfare and health of offenders" (p.575). It is essential for the OYA to continue to emphasis the agency's mission and philosophy at all levels of the organization. Youth welfare and reformation should remain a priority among the added obligation to support public safety. Otherwise, the culture of staff in charge of identification and referral for mental health treatment may be swayed towards one philosophy further hindering access to services to youth.

Concepts identified at the youth level included youth's behavioral observations, family history, culture and demographics. Staff discussed youth behavior type as a factor which they believe influence whether or not a youth received treatment. Negative 
behavioral observations were perceived to directly influence whether or not a youth will receive treatment. One facility staff member mentioned, "aggressive behavior hinders treatment." Age was also mentioned as a youth related factor. Younger youth were observed to get more negative attention from staff if behavior was negative. Staff reported particular observations including "staff tend to gravitate towards the younger youth.”

Factors identified at the staff level included staff's level of training, personality traits and the ability to engage in collaborative working relationships with mental health professionals as contributors to treatment allocation. Participants regarded general knowledge about mental health and treatment provided by the agency through "on the job training." Agency training provided in the statewide training academy was identified as the vehicle in which most staff receives specific knowledge about treatment. In addition, participants observed staff with previously acquired knowledge from past employment assisted staff in understanding the prioritization of treatment for youth by the agency. Additionally, staff mentioned the acquiring of specific mental health training is often times perceived as "on our own time" and at "staff expense." Overall, participants highlighted consistent themes describing the process of how education and knowledge is gained about mental health treatment, treatment availability and how it is allocated. Appelbaum and colleagues have published some work on the topic of mental health training for correctional officers (as cited in Parker, 2009, p. 643). Then need for correctional officer training is essential to their ability to participate in the identification and referral process. Custody and mental health staff often have different approaches and 
working environments leading to various perceptions of to those they serve. Interactions often include discussion which crossover philosophies. These discussions and interactions could be managed by having strong leadership or management present to navigate concerns on both sides. A more collaborative approach would support staff learning and development, ultimately lending to more positive outcomes. One area that was specifically identified was the potentially violent working conditions and the "differing professional cultures of security staff and mental health staff as major issues" (Parker, 2009, p. 643). Parker goes on to explain correctional officers "could and should be recognized as members of the multidisciplinary treatment team for offenders with mental illness, particularly on residential treatment units" (2009, p. 643). OYA utilizes multidisciplinary teams in their management of youth case plans. A sincere evaluation of the role and participation of these members would assist OYA in ensuring various perspectives are being provided throughout the service provision process. Findings suggest staff observe some limitations in this area. Involvement of non-clinical staff in MDTs would lend to a broader context for treatment team functionality. As OYA serves as a model to partnering juvenile justice agencies locally and throughout the country, these efforts may help to impact the JJS in its entirety. Next, implications from this study are outlined.

\section{Study Implications}

Among the many policy and practice implications from this study are potential recommendations for ongoing training for staff. Training in the areas of mental health symptoms, behavioral indicators and ways to support youth with mental health needs is 
needed on a more ongoing basis. Initial agency training was identified by staff as one of the exclusive times in their career where such valuable training was provided. Both correctional custody staff as well as mental health professionals should receive cross training on their roles so that non-mental health staff has a better understanding of ways they can support youth receiving treatment. Support for ongoing education and clinical supervision should be afforded as staff identified both the need and constraints for obtaining this after the initial training academy.

In terms of policy, it is suggested OYA implement means to routinely assess treatment availability to ensure quality services are meeting the mental health, trauma and substance abuse needs of youth. Among the various treatment types extracted from the data, nineteen different treatment groups and descriptions were identified. The majority of the treatment types included the use of Cognitive Behavioral approaches and was somewhat typical of correctional programming (i.e. offense specific treatment, vocational or skills based). Although this large variety illustrates the range of treatment groups and services, it demonstrates the need to formalize specific treatment approaches to match the needs of youth. Treatment classified in group formats included a wide variety of focus, with a limited number of the nineteen descriptions targeting mental health symptoms or trauma. Although OYA is under the guise of EBP, OYA would still stand to benefit from ongoing review and evaluation of the various treatment types implemented by providers within the agency. Providers are defined as those meeting the Oregon Administrative Rules QMHP standards and are certified to work as a QMHP in OYA facilities. They must hold both the education and experience demonstrating competency to provide 
mental health assessment, diagnosis and treatment (Oregon Administrative Rules, 2017). Implementing a framework to support agency providers in their efforts to sustain treatment provision is another important policy suggestion. Concepts such as how the need for treatment is identified, what types of treatment are offered, whether or not treatment services are limited to crisis intervention, individual or agency's capacity to provide specific treatment modalities, and level of clinical experience should be included in this framework.

Furthermore, policy and practice implications extend into areas of record keeping, evaluation and agency research. Efforts to enhance record keeping may assist the OYA with conducting a thorough analysis of treatment provision activities. This information could further support their efforts in fulfilling agency mission, goals, and legislative mandates to provide EBP. With a high prevalence of mental health and substance abuse disorders among youth, it is essential OYA demonstrate efforts to meet the needs of youth in custody. The lack of detailed electronic records may inhibit agency leadership and administrators who wish to advocate for budget and funding resources. Most importantly, this may impact their ability to provide effective mental health services and manage the services to ensure they meet standards of care.

Assessment, screening and identification efforts can be extended with improved data quality on treatment provision provided across diverse youth populations in custody. Furthermore, perceptions of organizational climate and culture, including leadership are an important area for resource allocation. 


\section{Limitations of the Study}

There are limitations to this study that should be mentioned. One, due to the exploratory nature of the study, there is a lack of extensive youth and staff interviews to fully understand the referral process that occurs in OYA facilities. Although data was used from JJIS, clinical and master facility files, valuable data that might provide more information pertaining to youth referral and treatment receipt was not readily available. Due to this reason, the extent to which this study could employ more rigorous quantitative analyses was more limiting and an unexpected outcome of the study. Unexpected limitations to accessing data included fragmented data sources illustrating treatment continuity as well as the location of clinic and facility files across the state. JJIS had limited data to serve as baseline observations and measurement for treatment allocation. Furthermore, JJIS had sparse data demonstrating the course of treatment provision for each individual youth. Available data on treatment provision was not accessible in a manner that was conducive to data mining. Some hard files could not be located due to the archiving process, which the files had not yet started. Some files were not accessible due to being in another individual's possession away from the archive building. Finally, some were not accessible due to the fact they were remaining at local county parole and probation offices awaiting transport to begin the archiving process. Two, the sample includes closed custody facilities and does not include camps or community residential facilities utilized by the OYA. The researcher felt youth characteristics would be substantially different in closed custody security facilities versus those of less secure camps. Three, a possible threat to the analysis of allocation of mental 
health treatment of youth offenders is present. Cultural implications for motivation and/or engagement in treatment were not measured in this study. This is an important aspect to highlight, as there exists a growing body of literature on race effects in the referral and provision of mental health and substance abuse services in the JJS. Given the small scale for literature on the allocation of treatment resources for youth offenders while in custody, this study serves as initial investigation into aspects of allocation. Future studies are needed to examine the issue of cultural differences in motivation to participate in treatment and may impact findings from this study. The researcher felt this limitation is acceptable given the scarcity of research on allocation of mental health resources in the JJS. Four, camps and closed custody facilities do not share the same facility characteristics and makeup. Five, along the same lines as the cultural limitation, data on cognitive abilities of youth offenders was not available in agency records. Cognitive limitations that impede an offender's ability to engage in treatment may pose a threat to the analysis and findings. Findings should be interpreted with the understanding of this limitation. Finally, camps and closed custody facilities have qualitatively different criteria for accepting youth. However, future analysis of such settings may be beneficial in adding to the knowledge of mental health service delivery in least restrictive settings, like those of OYA camps. In the future, a fair representation of juvenile justice entities in the OYA may need to include all facilities to examine referral and treatment receipt. Finally, this study is not generalizable to other partners in the JJS apart from OYA. OYA is unique to the Oregon criminal justice continuum and may have different resources, 
populations, and agency mission and values than other juvenile justice facilities. Interpretations from this study should be used with caution.

Another limitation to this study is the researcher's history as an OYA employee. Implicit bias may have impacted the lens and scope of understanding necessary to rigorously examine personal staff experiences. At the start of the study, the researcher was employed by the OYA, but throughout the course of the study separated from the agency. The amount of time lapsed since the onset of this study may have limited generalizability to the study's findings. OYA youth and staff populations may have significantly changed and improvements to treatment provision may have occurred at the time of this summary.

In conclusion, the methods selected for this study have both quantitative and qualitative aspects of a mixed methods design. Not only will they shed light on the convergence of sources of data, it will serve as a unique approach to the proposed investigation. The immense number of treatment categories, names, and recorded recommendations provided many challenges in categorizing and understanding the overall treatment provision process. A total of 19 various descriptions of treatment types were noted in youth files. Although there are limitations to this study, preliminary results and interpretations will be valuable for OYA as they will be more likely to be disseminated and understood by a larger audience. As all empirical research has limitations, the limitations in this study can be justified given the scope and intent of the study. 


\section{Areas for Future Research}

Given adolescent development and the fluidity of motivation in youth offenders, whether or not a youth is referred for treatment may also be explained by individual youth factors not explored in this study. Future research beyond the scope of this study is needed to investigate individual youth perceptions and hesitations for participating in treatment. Additionally, the categorization and description of treatment services can be better defined by agencies. For example, in a study completed by Fries et al. (2013), findings suggested numerous factors that may have influenced the number of adult prisoners receiving mental health services. Although the study was conducted with adult prisoners, the considerations for state level department of corrections record keeping are important to note. The study noted limitations in the Michigan Department of Corrections (MDOC) database in relation to the limited depth of mental health treatment records. Definitions of treatment and delineations of types of treatment also impacted the analysis. Treatment definitions may have not included crisis intervention, offender group programming, and services provided specifically by psychiatrists and psychologists. They identified, "it is possible that a person with dual diagnosis substance abuse and mental health issues could be receiving treatment for the substance abuse rather then mental health treatment" (Fries, et al., 2013, p. 323). State level government correctional agencies likely have similar mandates for offender record keeping. Both adult and juvenile agencies can learn from this limitation in record keeping. As OYA begins to prioritize and determine effective methods of recording mental health treatment and referral, the findings by Fries et al. (2013) may serve as an important reminder. Often, 
individuals are receiving multiple forms of treatment at one time. Ability to measure and record each is essential to evaluating services and areas for quality improvement. Further research is also needed to capture the specific predictors, which influence receipt of treatment. This study was limited in its ability to achieve a predictive analysis. Future research examining specific youth characteristics and their influence on receipt of treatment would further add to the literature described earlier in this study. Finally, as social workers and correctional staff work together to foster supportive environments for youth reformation, more research is needed in determine what aspects of an agency's social context support the allocation of treatment. This study aimed at exploring youth, staff, and agency/facility factors, which influence the receipt of treatment of youth in the OYA. It highlighted a significant number of findings, which further support the literature on environmental dynamics influencing treatment allocation. It used elements of social construction theory to test staff attitudes and perceptions of the treatment allocation process. In conclusion, this study can assist juvenile justice administrators, mental health professionals and juvenile correctional staff in improving the ways in which are identified and allocated mental health treatment. 


\section{References}

American Correctional Association (1991). Standards for juvenile training schools $\left(3^{\mathrm{rd}}\right.$ ed.). Laurel, MD: Author.

Andrews, D. A. \& Bonta, J. (2007). Risk-need-responsivity model for offender assessment and Rehabilitation. Ottawa: Correctional Services of Canada.

Archer, R., Stredny, R., Mason, J., \& Arnau, R. (2004). An examination and replication of the psychometric properties of the Massachusetts Youth Screening Instrument-Second Edition (MAYSI) among adolescents in detention settings. Assessment, 11(4), 290-302.

Archer, R., Bisbee, E., Spiegel, D., Handel, R., \& Elkins, D. (2010). Validity of the Massachusetts Youth Screening Instrument-2 (MAYSI) scales in juvenile justice settings. Journal of Personality Assessment, 92(4), 337-348.

Ashford, J., Sales, B., \& Reid, W. (2001). Treating adult and juvenile offenders with special needs. Washington, DC: American Psychological Association.

Atkins, L., Pumariega, A., M.D., Rogers, K., Montgomery, L., Nybro, C., Jeffers, G., \& Sease, F. (1999). Mental health and incarcerated youth. I: Prevalence and nature of psychopathology. Journal of Child and Family Studies, 8(2), 193-204.

Barton, W. (2006). Incorporating the strengths perspective into intensive juvenile aftercare. Western Criminology Review, 7(2), 48-61.

Blueprint for Change: A Comprehensive Model for the Identification and Treatment of Youth with Mental Health Needs in Contact with the Juvenile Justice System (2006). Retrieved from http://www.modelsforchange.net. 
Boesky, L. (2001). Mental health training in juvenile justice: A necessity.

Corrections Today, 63, 1-6.A

Boyatzis, R. (1998). Transforming qualitative information: Thematic analysis and code development. Thousand Oaks, Sage Publications, Inc.

Breda (2003). Offender ethnicity and mental health service referrals from juvenile courts. Criminal Justice and Behavior, 30(6), 644-667.

Caeti, T., Hemmens, C., Cullen, F., \& Burton, V. (2003). Management of Juvenile Correctional Facilities. The Prison Journal, 83, 383-405.

Callahan, L. (2004). Correctional officer attitudes toward inmates with mental disorders. International Journal of Forensic Mental Health, 3(1), 37-54.

Cauffman, E. (2004). A statewide screening of mental health symptoms among juvenile offenders in detention. Journal of the American Academy of Child and Adolescent Psychiatry, 43(4), 430-439.

Cohen, P. (1993). Demographic factors in the use of children's mental health services. American Journal of Public Health, 83(1), 49-52.

Coleman, D. (2005). Trauma and incarcerated youth. Journal of Evidenced-Based Social Work, 2 (3/4), 113-124.

Corrigan, P. \& Watson, A. (2003). Factors that explain how policy makers distribute resources to mental health services. Psychiatric Services, 54 (4), 501-507.

Corrigan, P., Watson, A., Warnpinksi, A. \& Gracia, G. (2004). Stigmatizing attitudes about mental health illness and allocation of resources to mental health services. Community Mental Health Journal, 40 (4), 297-307. 
Cocozza, J., Hartsone, E., \& Braff, J. (1981). Mental health treatment of violent juveniles: An assessment of need. Crime and Delinquency, 27 (4), 487-496.

Cocozza, J. \& Skowyra, K. (2000). Youth with Mental Health Disorders: Issues and Emerging Responses. Juvenile Justice, 7(1). Retrieved from http://www.ncjrs.org/html/ojjdp/jjjnl_2000_4/youth.html.

Creswell, J., Fetters, M., and Ivankova, N. (2004). Designing a mixed methods study in primary care. Anals of Family Medicine, 2(1), 7-12.

Derogatis, L., \& Melisaratos, N. (1983). The Brief Symptom Inventory: An introductory report. Psychological Medicine, 13, 595-605.

Diwan, S. (1999). Allocation of case management resources in long-term care: Predicting high use of case management time. The Gerontologist, 39 (5), 580-590.

Driscoll, D., Appiah-Yeboah, A., Salib, P., \& Rupert, D. (2007). Merging qualitative and quantitative data in mixed methods research: How to and why not. Ecological and Environmental Anthropology, 3(1), 19-28.

Duffee, D \& Carslon B. (1996). Competing value premise for the provision of drug treatment to probationers. Crime \& Delinquency, 42, 574-592.

Farrell, J., Young, D., \& Taxman, F. (2011). Effects of organizational factors on use of juvenile supervision practices. Criminal Justice and Behavior, 38(6), 565-583.

Foglia, M., Pearlman, R., Bottrell, M., Altemose, J., \& Fox, E. (2007). Priority setting and the ethics of resource allocation within VA healthcare facilities: Results of a survey. Organizational Ethics, Fall/Winter, 83-96.

Fraser, K. \& Estabrooks, C. (2008). What factors influence case manager's resource 
allocation decisions? A systematic review of the literature. Medical Decision Making, 28, 394-410.

Fries, B., Schmorrow, A., Lang, S., Margolis, P., Heany, J., Brown, G., Barbaree, H., \& Hirdes, J. (2013). International Journal of Law and Psychiatry, 36, 316-325.

Furnham, A. \& McClelland, A. (2004). The allocation of scarce resources: Social housing. Social behavior and personality, 32 (1), 45-54.

Garland, A., et al. (2005). Racial and ethnic differences in utilization of mental health services among high-risk youths. American Journal of Psychiatry, 162 (7), 13361343

Glisson, C. \& Green, P. (2006). The effects of organizational culture and climate on the access to mental health care in child welfare and juvenile justice systems. Administration and Policy in Mental Health and mental Health Services Research, 33, 433-448.

Green, A., Albanese, B., Cafri, G., \& Aarons, G. (2014). Leadership, organizational climate, and working alliance in a children's mental health service system. Community Mental Health Journal, 50, 771-777.

Grisso, T., \& Barnum, R. (2006). Massachusetts Youth Screening InstrumentVersion 2: User's manual and technical report (Rev. ed.). Sarasota, FL: Professional Resource Press.

Grisso, T.,\&Quinlan, J. C. (2005). Massachusetts Youth Screening InstrumentVersion 2. In T. Grisso, G. Vincent, \& D. Seagrave (Eds.), Mental health screening and assessment in juvenile justice (pp. 99-111). New York, NY: 
Guilford.

Herz, D. (2001). Understanding the use of mental health placements by the juvenile justice system. Journal of Emotional and Behavioral Disorders, 9 (172), 172-181.

Hubbard, D. (2007). Getting the most out of correctional treatment: Testing the responsivity principles on male and female offenders. Federal Probation, 71(1), $2-8$.

Hubbard, D. \& Mattews, b. (2008). Reconciling the differences between the "gender responsive" and the "what works" literatures to improve services for girls. Crime and Delinquency, 54 (2), 225-258.

Kelly, W., Macy, T. \& Mears, D. (2005). Juvenile referrals in Texas: An assessment of Criminogenic needs and the gap between needs and services. The Prison Journal, $85,467-489$.

Krejcie, R. V., \& Morgan, D. W. (1970). Determining sample size for research activities. Educational and Psychological Measurement, 30, 607-610.

LeCroy, C., Stevenson, P., \& MacNeil, G. (2001). Systems considerations in treating juvenile offenders with mental disorders. In J.B. Ashford, B.D. Sales, \& W.H.

Reid (Eds.), Treating adult and juvenile offenders with special needs (pp. 401-417).

Washington, DC: American Psychological Association.

Levin, I. \& Chaprman, D. (1993). Risky decision making and allocation of resources for Leukemia and AIDS programs. Health Psychology, 12 (2), 110-117.

Maschi, T., Schwalbe, C., Morgen, K., Gibson, S., \& Violette, N. (2009). Exploring the influence of gender on adolescents' service needs and service pathways. Children 
and Youth Services Review, 31, 257-264.

Mason, T. \& Mercer, D. (1999). The sociology of the mentally disordered offender. New York: Pearson Education .

Maill, C. (1996). The social construction of adoption: Clinical and community perspectives. Family Relations, 45(3), 309-317.

Mental Health and Juvenile Justice Collaborative for Change. (2014). Better Solutions for youth with mental health needs in the juvenile justice system [White Paper]. Retrieved from https://www.ncmhjj.com/wpcontent/uploads/2014/01/WhitepaperMental-Health-FINAL.pdf.

Mitchell, O., MacKenzie, D.L., Gover, A.R., \& Styve, G.J. (2001). The influences of personal Background on perceptions of juvenile correctional environments. Journal of Criminal Justice, 29, 67-76.

Moak, S.C. \& Wallace, L.H. (2000). Attitudes of Louisiana practitioners toward rehabilitation of juvenile offenders. American Journal of Criminal Justice, 24, 271-285.

Models for Change (2006). Mental Health Screening within Juvenile Justice: The Next Frontier. National Center for Mental Health and Juvenile Justice. Retrieved from http://www.modelsforchange.net.

Moos, R. Nichol, A. \& Moss, B. (2002). Global assessment of functioning ratings and the allocation and outcomes of mental health services. Psychiatric Services, 53 (6), 730-737. 
Morgan, D. (1997). (2 ${ }^{\text {nd }}$ ed). Focus groups as qualitative research. Thousand Oaks: Sage Publications.

Oregon Administrative Rules (2017). Oregon Youth Authority Mental Health Certification Standards. Oregon Administrative Rules, chapter 416, division 70, rule 0030 . Retrieved from http://arcweb.sos.state.or.us/pages/rules/oars_400/oar_416/416_070.html.

Oregon Youth Authority Agency Website. http://www.oregon.gov/OYA/pso.shtml. Retrieved on August 23, 2011.

Oregon Youth Authority (2007). New commitments to OYA legal custody (B: Intake 20). Retrieved from http://www.oregon.gov/OYA/policies/III-B-2.0.pdf.

Oregon Youth Authority (2008). Mental Health Gap Survey. Oregon Youth Authority state agency website. Retrieved from http://www.oregon.gov/OYA/docs/MentalHealthGap_2008.pdf.

Oregon Youth Authority at a Glance (January 2010). Retrieved from http://www.oregon.gov/OYA/docs/OYA_at_a_glance_Web.pdf.

Oregon Youth Authority (2011). OYA at a Glance. Oregon Youth Authority state agency website. Retrieved from http://www.oregon.gov/OYA/docs/OYA at a glance Web.pdf?ga=t.

Oregon Youth Authority Career Opportunities (May 2011). Retrieved from http://agency.governmentjobs.com/oregon/default.cfm?\&searchApplied=1\&DEP $\underline{\text { ARTMENTID }=16004,18229,18549 .}$.

Oregon Youth Authority (2016). OYA Biopyschosocial Summary. Oregon Youth 
Authority state agency website. Retrieved from http://www.oregon.gov/oya/docs/2016-YouthBiopsychosocialSummary.pdf.

Oregon Youth Authority (2017). OYA Quick Facts. Oregon Youth Authority state agency website. Retrieved from http://www.oregon.gov/oya/docs/QuickFacts/QuickFacts-Jan2017.pdf.

Osman, A., Gutierrez, P., Barrios, F., Bagge, C., Kopper, B., \& Linden, S. (2005). The Inventory of Suicide Orientation-30: Further validation with adolescent psychiatric inpatients. Journal of Clinical Psychology, 61(4), 481-497.

Pallant, J. (2005). ( $2^{\text {nd }}$ ed). SPSS Survivial Manual: A step by step guide to using data analysis using SPSS Version 12.0. Chicago: Mcgraw-Hill.

Parker, G. (2009). Impact of a mental health training course for correctional officers on a special housing unit. Psychiatric Services, 60(5), 640-645,

Payne, M. (1991). Modern social work theory: A critical introduction. London: The Macmillan Press.

Peterjl-Taykor, C. (1999). Care of individuals in correctional facilities. In C. Glod (Ed.), Contemporary psychiatric-mental health nursing: The brain-behavior connection (pp. 620-633). Philadelphia: FA Davis.

Peterson, R. \& Palumbo, D. (1997). The social construction of intermediate punishments. The Prison Journal, 77(1), 77-91.

Pullman, M., Kerbs, J., Korloff, N., Veach-White, E., Gaylor, R., \& Sieler, D. (2006). Juvenile offenders with mental health needs: Reducing recidivism using wraparound. Crime and Delinquency, 52, 375-396. 
Pumariega, A., Atkins, L., Rogers, K., M.D., Montgomery, L., Nybro, C., Caesar, R., \& Millus, D. (1999). Mental health and incarcerated youth. II: Service utilization. Journal of Child and Family Studies, 8(2), 205-215.

Quinn, J. \& Gould, L. (2003). The prioritization of treatment among Texas parole officers. The Prison Journal, 83(3), 323-336.

RAND Health Research Highlights (2001). Mental health care for youth. Who gets it? How much does it cost? Who pays? Where does the money go? Retrieved from http://www.rand.org/pubs/research_briefs/RB4541/index1.html.

Rawal, P., Romansky, J., Jenuwine, M., \& Lyons, J. (2004). Racial differences in the mental health needs and service utilization of youth in the juvenile justice system. The Journal of Behavioral Health Services and Research, 31(3), 242-254.

Rogers, K., Zima, B., Powell, E., \& Pumariega, J. (2002). Who is referred to mental health services in the juvenile justice system? Journal of Child and Family Studies, 10(4), 485- 494.

Rosenthal, J. (1996). Qualitative descriptors of strength of association and effect size. Journal of Social Sciences Research, 21(4), 37-59.

Scheider, A. \& Ingram, H. (1993). Social construction of target populations: Implications for politics and policy. American Political Science Review, 87(2), 334-347.

Scott, E. \& Steinburg, L. (2008). Rethinking juvenile justice. Cambridge: Harvard University Press.

Shelton, D. (1999). Estimates of emotional disorder in detained and committed youth. (Final Report). Baltimore, MD: Maryland Department of Juvenile Justice. 
Shufelt, J. \& Cocozza, J. (2006). Youth with mental health disorders in the juvenile justice system: Results from a multi-state prevalence study. Delmar: NY. National Center for Mental Health and Juvenile Justice.

Snyder, H. \& Sickmund, M. (1995). Juvenile offenders and victims: A national report. Washington, DC: Office of Juvenile Justice and Delinquency Prevention.

Snyder, H. \& Sickmund, M. (2006). Juvenile Offenders and Victims: 2006 national report. Washington, DC: U.S. Department of Justice, Office of Justice Programs, Office of Juvenile Justice and Delinquency Prevention.

Spinney, E., Yeide, M., Feyerherm, W., Cohen, M., Stephenson, R., \& Thomas, C. (2016). Racial disparities in referrals to mental health and substance abuse services from the juvenile justice system: A review of the literature. Journal of Crime and Justice, 39(1), 153-173.

Tabachnick, B. \& Fidell, L. (2007). Using multivariate statistics $\left(5^{\text {th }}\right.$ ed). Boston: Pearson Ecuation.

Tashakkoru, A. \& Creswell, J. (2007). Editorial: Exploring the nature of research questions in mixed methods research. Journal of Mixed Methods Research, 1(3), 207-211.

Taxman, F., \& Bouffard, J. (2003). Substance abuse counselor's treatment philosophy and the content of the treatment services provided to offenders in drug court programs. Journal of Substance Abuse and Treatment, 33, 201-209.

Taxman, F., Cropsey, K., Melnick, G., \& Perdoni, M. (2008). COD services in 
community correctional settings: An Examination of organizational factors that affect service delivery. Behavioral Sciences and the Law, 26, 435-455.

Teplin, L., Abram, K., McClelland, G., Dulcan, M., \& Mericle, A. (2002). Psychiatric disorders in youth in juvenile detention. Archives of General Psychiatry, 59, 1133-1143.

Teplin, L., Abram, K., McClelland, G., Washburn, J., \& Pikus, A. (2005). Detecting mental disorder in juvenile detainees: Who receives services? American Journal of Public Health, 95, 1773-1780.

Thompson, M., Newell, S., \& Carlson, M. Race and access to mental health and substance abuse treatment in the criminal justice system. Journal of Offender Rehabilitation, (55)2, 69-94.

Turner, F. J. (Ed.). (1986). Social work treatment: Interlocking theoretical perspectives ( $3^{\text {rd }}$ ed.). New York: Free Press.

Ventura, J., Liberman, R., Green, M., Shaner, A., \& Mintz, J. (1998). Training and quality assurance with the structured clinical interview for DSM-IV (SCID-I/P). Psychiatry Research, 79, 163-173.

Wasserman, G., Ko, S., \& McReynolds, L. (2004). Assessing the mental health status of youth in juvenile justice settings. Juvenile Justice Bulletin (August): 1-7.

Wong, C. (2001). Chemical castration: Oregon's innovative approach to sex offender rehabilitation, or unconstitutional punishment? Oregon Law Review, 80(1).

Yan, J. \& Dannerbeck, A. (2011). Exploring the relationship between gender, mental 
health needs, and treatment orders in a metropolitan juvenile court. Journal of Child and Family Studies, 20(9), 9-22.

Young, D., Farrell, J., Henderson, C. \& Taxman, S. (2009). Filing service gaps:

Providing intensive treatment services for offenders. Drug and Alcohol Dependence, 103S, S33-S42. 


\section{Appendix A: Case File Recording Form}

\begin{tabular}{|c|c|c|c|c|c|c|}
\hline & $\mathrm{A}$ & $\mathrm{B}$ & $\mathrm{C}$ & $\mathrm{D}$ & $\mathrm{E}$ & $\mathrm{F}$ \\
\hline $\begin{array}{l}\text { Diagnosis (check if } \\
\text { present) }\end{array}$ & $\begin{array}{l}\text { Initial } \\
\text { Psychiatric }\end{array}$ & $\begin{array}{l}\text { Last } \\
\text { Psychiatric }\end{array}$ & $\begin{array}{l}\text { Initial } \\
\text { Psychological by } \\
\text { Intern- }\end{array}$ & $\begin{array}{l}\text { Initial } \\
\text { Psychological by } \\
\text { Contractor }\end{array}$ & $\begin{array}{l}\text { Last } \\
\text { Psychological by } \\
\text { Intern }\end{array}$ & $\begin{array}{l}\text { Last Psychological } \\
\text { by Contractor }\end{array}$ \\
\hline \multicolumn{7}{|l|}{ Adjustment Disorder } \\
\hline \multicolumn{7}{|l|}{$\begin{array}{l}\text { Anxiety Disorder } \\
\text { (types) }\end{array}$} \\
\hline \multicolumn{7}{|l|}{ Acute Stress Disorder } \\
\hline \multicolumn{7}{|l|}{ Agoraphobia } \\
\hline \multicolumn{7}{|l|}{$\begin{array}{l}\text { Generalized Anxiety } \\
\text { Disorder }\end{array}$} \\
\hline \multicolumn{7}{|l|}{$\begin{array}{l}\text { Obsessive Compulsive } \\
\text { Disorder }\end{array}$} \\
\hline \multicolumn{7}{|l|}{ Panic Disorder } \\
\hline \multicolumn{7}{|l|}{ Phobia } \\
\hline \multicolumn{7}{|l|}{ PTSD } \\
\hline \multicolumn{7}{|l|}{ Eating Disorders } \\
\hline \multicolumn{7}{|l|}{ Anorexia Nervosa } \\
\hline \multicolumn{7}{|l|}{ Bulimia Nervosa } \\
\hline \multicolumn{7}{|l|}{$\begin{array}{l}\text { Impulse Control } \\
\text { Disorders }\end{array}$} \\
\hline \multicolumn{7}{|l|}{$\begin{array}{l}\text { Intermittent Explosive } \\
\text { Disorder }\end{array}$} \\
\hline \multicolumn{7}{|l|}{ Trichotillomania } \\
\hline \multicolumn{7}{|l|}{ Mood Disorders } \\
\hline \multicolumn{7}{|l|}{ Bipolar } \\
\hline \multicolumn{7}{|l|}{ Cyclothymic } \\
\hline \multicolumn{7}{|l|}{ Dysthymic } \\
\hline \multicolumn{7}{|l|}{ Major Depression } \\
\hline \multicolumn{7}{|l|}{ Psychotic Disorders } \\
\hline \multicolumn{7}{|l|}{ Schizoaffective } \\
\hline \multicolumn{7}{|l|}{ Schizophrenia } \\
\hline \multicolumn{7}{|l|}{ Schizophreniform } \\
\hline \multicolumn{7}{|l|}{$\begin{array}{l}\text { Brief Psychotic } \\
\text { Disorder }\end{array}$} \\
\hline Substance Abuse & & & & & & \\
\hline Substance Abuse & & & & & & \\
\hline (specify substance) & & & & & & \\
\hline Substance Dependenc & & & & & & \\
\hline (specify substance) & & & & & & \\
\hline Personality Disorders & & & & & & \\
\hline Borderline & & & & & & \\
\hline Anti-Social & & & & & & \\
\hline Narcissistic & & & & & & \\
\hline Histrionic & & & & & & \\
\hline Other (Specify) & & & & & & \\
\hline Other (Specify) & & & & & & \\
\hline No Diagnosis Given & & & & & & \\
\hline GAF & & & & & & \\
\hline CGAS & & & & & & \\
\hline
\end{tabular}

\begin{tabular}{|c|c|c|}
\hline $\begin{array}{c}\text { MAYSI Domains } \\
\text { *Warning or Caution Ranges } \\
\text { (check if present) }\end{array}$ & Initial Intake MAYSI & Last Intake MASYI \\
\hline Alcohol and Drug & & \\
\hline Angry/Irritable & & \\
\hline Depressed/Anxious & & \\
\hline Somatic Complaints & & \\
\hline Suicidal Ideation & & \\
\hline $\begin{array}{c}\text { Thought Disturbances } \\
\text { (Boys Only) }\end{array}$ & & \\
\hline Traumatic Experiences (Girls) & & \\
\hline
\end{tabular}


Behavioral Indicators

Youth Incident Reports (within time of intake):

Within 6 months

Within 12 months

Within 24 months

Within 36 months

\begin{tabular}{|l|l|l|l|l|l|}
\hline IQ Over 80 & & IQ 70-80 & & IQ Below 70 & IQ Score \\
\hline
\end{tabular}

history:___ no suicide attempt in the last 3 years

1 suicide attempt in the last 3 years

Suicide Attempt years

Treatment Received

Previously assessed (prior to OYA) for treatment:

yes

no

unknown

no info in file

type of Tx

Recom/Received

Type of previous treatment:

inpatient

outpatient

other

Time treatment (assessment) occurred:

within 6 months

within 12 months

within 24 months other (older)

Initial Assessment:

in need of tx

not in need of $\mathrm{tx}$

unknown

Subsequent assessment:

in need of $\mathrm{tx}$

not in need of tx

unknown

Previous psychiatric hospitalization: none 1 within 12 months

1 within 24 months 1 more than 2 years

\begin{tabular}{|c|c|c|c|c|c|c|c|c|c|c|}
\hline TX RECOM & $\mathrm{A}$ & $\mathrm{B}$ & $\mathrm{C}$ & $\mathrm{D}$ & $E$ & $\mathrm{~F}$ & Intake MSA by Q & $\begin{array}{c}\text { ARB/MDT } \\
\text { Rec }\end{array}$ & $\begin{array}{c}\text { RECEVED? } \\
\mathrm{Y} / \mathrm{N}\end{array}$ & $\begin{array}{l}\text { @ WHICH } \\
\text { FACILITY }\end{array}$ \\
\hline \multicolumn{11}{|l|}{ CBT } \\
\hline \multicolumn{11}{|l|}{ Seeking Safety } \\
\hline \multicolumn{11}{|l|}{ Trauma } \\
\hline \multicolumn{11}{|l|}{ Indiv Co } \\
\hline \multicolumn{11}{|l|}{ ART } \\
\hline \multicolumn{11}{|l|}{ Anger } \\
\hline \multicolumn{11}{|l|}{ ATOD } \\
\hline \multicolumn{11}{|l|}{ CWD } \\
\hline \multicolumn{11}{|l|}{$\begin{array}{l}\text { Med Rev (see } \\
\text { Psych MD) }\end{array}$} \\
\hline \multicolumn{11}{|l|}{ DBT } \\
\hline \multicolumn{11}{|l|}{ Skills } \\
\hline \multicolumn{11}{|l|}{ COG Skills } \\
\hline \multicolumn{11}{|l|}{ HR } \\
\hline \multicolumn{11}{|l|}{$\begin{array}{l}\text { Psych MD } \\
\text { Med F/U in }\end{array}$} \\
\hline \multicolumn{11}{|l|}{ Other } \\
\hline \multicolumn{11}{|l|}{ Other } \\
\hline \multicolumn{11}{|l|}{ No Recom } \\
\hline $\begin{array}{l}\text { Placement } \\
\text { Recom Only }\end{array}$ & & & & & & & & & & \\
\hline
\end{tabular}




\section{Appendix B: Focus Group Questions and Survey Items}

1. Tell us your name and how long you have been working for the agency and what your current position is.

2. How was it that you first learned about mental health treatment and other treatment resources for youth offenders?

3. What in particular, do you feel influences whether or not mental health treatment is allocated to youth offenders?

4. One of the things that we are especially interested in is how youth factors influence the decision to allocate mental health treatment to youth. What can you tell us about that?

5. In addition to youth factors that you have identified, what staff factors influence allocation of mental health treatment resources?

6. Finally, what facility/program or agency factors influence allocation of mental health treatment to youth offenders?

7. In thinking about your particular facility, how often are you trained?

8. Does your facility undergo reductions and changes often?

9. Does your facility offer mental health treatment?

10. One of the concepts we are really interested in exploring is position of power and how power can influence decision-making. Your responses to these questions are merely for discussion purposes to initiate conversation and dialogue. Using the Likert scale provided for the next five questions (strongly agree-slightly agree-neutral- 
slightly disagree-strongly disagree) do you feel you have a say in whether or not a youth receives treatment?

11. Again using the Likert scale....Do you feel you have enough opportunity to voice your input in the decision making process?

12. Again using the Likert scale....In your perception, do you believe a youth's parole officer has the most amount of power and influence in the decision to allocate treatment to youth?

13. Again using the Likert scale....Do you agree that youth should be included in the decision making process?

14. Again using the Likert scale....Do you believe decisions are made without input from others involved in a youth's case?

15. Is there anything else we need to know about factors that influence allocation of mental health treatment to youth offenders?

16. Is there anything else we should have talked about, but didn't?

Date you attended focus group:

Gender (please check): Male: Female:

Racelethnicity: (please check one):

White/Caucasian:

African American:

Hispanic:

Native American:

Asian:

Other (please note):

Staff position (please check one): 
Group Life Coordinator

Youth Corrections Unit Coordinator

Qualified Mental Health Professional:
Treatment Manager:

Facility Superintendent:

Program Director: 